\title{
Comparison of moving and fixed basis sets for nonadiabatic quantum dynamics at conical intersections
}

\author{
Evgenii Titov, Alexander Humeniuk, and Roland Mitrić \\ Institut für Physikalische und Theoretische Chemie, Julius-Maximilians-Universität \\ Würzburg, Emil-Fischer-Strasse 42, 97074 Würzburg, Germany. \\ E-mail: roland.mitric@uni-wuerzburg.de
}

\begin{abstract}
We assess the performance of two different types of basis sets for nonadiabatic quantum dynamics at conical intersections. The basis sets of both types are generated using Ehrenfest trajectories of nuclear coherent states. These trajectories can either serve as a moving (timedependent) basis or be employed to sample a fixed (time-independent) basis. We demonstrate on the example of two-state two-dimensional and three-state five-dimensional models that both basis set types can yield highly accurate results for population transfer at intersections, as compared with reference quantum dynamics. The details of wave packet evolutions are discussed for the case of the two-dimensional model. The fixed basis is found to be superior to the moving one in reproducing nonlocal spreading and maintaining correct shape of the wave packet upon time evolution. Moreover, for the models considered, the fixed basis set outperforms the moving one in terms of computational efficiency.
\end{abstract}

\section{Keywords}

nonadiabatic quantum dynamics, multiconfigurational Ehrenfest, basis set, conical intersection 


\section{Introduction}

Intersections and near-intersections of potential energy surfaces (PESs) can occur in polyatomic molecules ${ }^{1,2}$ and lead to ultrafast nonradiative decay of electronically excited states. ${ }^{3-6}$ Often the nonradiative electronic transitions may be accompanied with remarkable nuclear dynamics ${ }^{7-12}$ the isomerization of molecular photoswitches being a typical example. ${ }^{13-16}$

A prototypical situation of intersections between PESs in polyatomic molecules is the so-called conical intersection (CoIn) of two PESs. ${ }^{17}$ The intersection is called "conical" since the difference of the two intersecting PESs in the vicinity of the intersection is a cone in the space spanned by energy and two directions forming a so-called branching space, the space where the degeneracy is lifted. (This is true if the nuclear gradients of the diabatic energy difference and of the coupling are non-zero at the nuclear configuration corresponding to the intersection. ${ }^{17}$ ) Many studies were devoted to investigation of CoIns and their role in photochemistry. ${ }^{18-20}$

A somewhat more exotic situation is the simultaneous intersection of three PESs. ${ }^{21-24}$ In general, the branching space for such three-state intersections is five-dimensional, and not two-dimensional as for CoIns (of two states). ${ }^{21,22}$ We note here that the term "conical intersection" is often used for the three-state intersections as well, apparently due to historical reasons, though the shape of the energy difference in the space spanned by energy and five branching space vectors is not a cone. In what follows we will keep using simply the term "intersection" for the three-state case.

The description of dynamics at conical intersections and in cases of near-degeneracies requires multistate electronic treatment, since several electronic states are involved in the (near-)intersection and nonadiabatic processes are expected to occur. ${ }^{25}$ In general, the two major classes of methods for modeling nonadiabatic molecular dynamics are quantum ${ }^{26}$ and mixed quantum-classical approaches, ${ }^{27}$ both having certain advantages and disadvantages. We also note that purely classical description of electron and nuclear dynamics may also yield reasonable results (at least for an one-electron system). ${ }^{28}$

The quantum propagation on a grid, the straightforward approach to quantum dynamics, being numerically exact is subjected to a socalled "exponential curse", i.e. the number of grid points needed to cover a volume of interest rises as $N^{d}$, with the number of grid points 
per dimension $N$ and the number of dimensions $d$. Therefore, the propagation on a grid becomes unfeasible for systems with many degrees of freedom (DoFs). The mixed quantum-classical methods, such as the widely used surface hopping approach, ${ }^{29,30}$ are much more efficient, but on the downside lack quantum effects of nuclear motion. The multiconfiguration time-dependent Hartree (MCTDH) $)^{31,32}$ and its multi-layer (ML) extension (ML-MCTDH) ${ }^{33,34}$ allow one to account for many degrees of freedom and treat nuclei quantum-mechanically, but still the grids over which the single-particle functions are expanded are necessary.

Methods which use trajectories in the phase space corresponding to nuclear DoFs have emerged for the description of multidimensional multistate quantum dynamics, allowing one to account for nuclear quantum effects, occurring in particular during nonadiabatic processes. Among these methods are the Frozen Gaussian method by Heller, ${ }^{35}$ Gaussian-based multiconfiguration time-dependent Hartree (G-MCTDH) ${ }^{36,37}$ and its all-Gaussian variant, also known as the variational multi-configurational Gaussian (vMCG) with its direct dynamics (DD) extension (DD-vMCG), ${ }^{38}$ full multiple spawning (FMS) ${ }^{39,40}$ and its ab-inito (AI) variant (AIMS), ${ }^{41}$ Multiconfigurational Ehrenfest (MCE) including different formulations (versions 1 and 2) MCEv1 ${ }^{42} / \mathrm{MCEv}^{43}$ as well as AI-MCE ${ }^{44}$ and its implementation with time-dependent diabatic basis (TDDB) MCE-TDDB,${ }^{45}$ surface hopping Gaussians (SHG), ${ }^{46}$ surface hopping coupled coherent states (SH-CCS), ${ }^{47}$ AI multiple cloning $(\mathrm{AIMC})^{48}$ and its TDDB implementation (AIMC-TDDB), ${ }^{49}$ pseudospectral Gaussian dynamics, ${ }^{50}$ the Ehrenfest method with fully quantum nuclear motion (Qu-Eh), ${ }^{51}$ and dynamics with multiple Davydov ansatz. ${ }^{52}$ All of them have in common the use of time-dependent basis sets for description of time-dependent nuclear wave function. We also note that the trajectory-based methods may be used not only for nonadiabatic problems but also for description of tunneling (e.g., the CCS method $^{53}$ ) and system-bath dynamics (e.g., the local coherent-state approximation (LCSA) $\operatorname{method}^{54}$ ).

Recently, Saller and Habershon proposed to utilize trajectories in nuclear phase space to sample from them a fixed, time-independent basis set, arguing that the trajectories span a part of the volume which is relevant for quantum dynamics, and therefore provide the suitable fixed basis functions. ${ }^{55}$ This approach represents a "middle road" between dynamics on a grid from one side and dynamics with a moving basis from the other. 
Here, we compare the performance of two types of basis sets (moving vs. fixed) for the description of nonadiabatic dynamics at the two-state and three-state model intersections. To describe dynamics we use a method which employs Ehrenfest trajectories of nuclear coherent states. This method is very similar to the multiconfigurational Ehrenfest method of Shalashilin (specifically to MCEv2, ${ }^{43,56}$ i.e. MCE with independent from each other trajectories), when the time-dependent basis set is used. However, the technical details of derivation and implementation differ from those of the MCE.

We demonstrate by comparing with reference benchmark calculations (dynamics on a grid for the two-state two-dimensional model and MCTDH for the three-state five-dimensional model) that both types of basis sets yield highly accurate results, specifically for the description of population transfer. The detailed analysis of the wave packet evolutions for the two-dimensional model reveals that the fixed basis set outperforms the time-dependent one in describing spreading of the wave packet with time, thus taking better account of nonlocality, and in maintaining the correct shape of the nuclear wave function during propagation. Moreover, for the studied model problems, the fixed basis set is found to be computationally more efficient than the moving one.

\section{Theory and computation}

\subsection{Working equations}

The time evolution of a quantum pure molecular state $|\Psi\rangle$ is described by the time-dependent Schrödinger equation:

$$
\mathrm{i} \hbar|\dot{\Psi}\rangle=\hat{H}|\Psi\rangle
$$

where $\hat{H}$ is a molecular Hamiltonian, represented as

$$
\hat{H}=\sum_{A, B} \hat{H}_{A B}|A\rangle\langle B|
$$

Here, $A$ and $B$ label the electronic diabatic orthonormal states and $\hat{H}_{A B}$ are nuclear operators.

The total molecular state is expanded in a basis of direct products

of nuclear coherent states $(\mathrm{CSs})\left|\mathbf{z}_{n}^{(A)}(t)\right\rangle$, which can be made time- 
dependent, and electronic states $|A\rangle$ :

$$
|\Psi(t)\rangle=\sum_{A, n} a_{n}^{(A)}(t)\left|\mathbf{z}_{n}^{(A)}(t)\right\rangle|A\rangle
$$

In general, an arbitrary number of electronic states as well as coherent states can be used in this expansion. Further, we set

$$
\mathbf{z}_{n}^{(A)}=\mathbf{z}_{n} \quad \forall A
$$

i.e., the coherent states are mirrored on all electronic states. This setup is called a single-set formulation, in contrast to the multi-set formulation for which different electronic states bear different sets of coherent states $\left\{\mathbf{z}_{n}^{(A)}\right\} .57$ The single set formulation is a natural choice when Ehrenfest trajectories are employed, since all electronic states are involved in propagation of a single trajectory, and this formulation was reported to provide a better description of nonadiabatic dynamics. ${ }^{55}$ The multidimensional coherent state $\left|\mathbf{z}_{n}\right\rangle$ is a direct product of one-dimensional coherent states corresponding to single nuclear DoFs $\left|z_{n}^{(f)}\right\rangle: 58$

$$
\left|\mathbf{z}_{n}\right\rangle=\prod_{f}^{\substack{\text { number of } \\ \text { nucl. DoFs }}}\left|z_{n}^{(f)}\right\rangle
$$

The position representation of the latter (for the nuclear DoF $Q_{f}$ ) is the Gaussian function: ${ }^{59}$

$z_{n}^{(f)}\left(Q_{f}\right)=\left\langle Q_{f} \mid z_{n}^{(f)}\right\rangle=\left(\frac{\gamma_{n}^{(f)}}{\pi}\right)^{\frac{1}{4}} \exp \left(-\frac{\gamma_{n}^{(f)}}{2}\left(Q_{f}-q_{n}^{(f)}\right)^{2}+\frac{\mathrm{i}}{\hbar} p_{n}^{(f)}\left(Q_{f}-q_{n}^{(f)}\right)+\frac{\mathrm{i} p_{n}^{(f)} q_{n}^{(f)}}{2 \hbar}\right)$

with parameters $q_{n}^{(f)}$ and $p_{n}^{(f)}$ being the position and momentum of a Gaussian center, and $\gamma_{n}^{(f)}$ being the width parameter. The $z_{n}^{(f)}\left(Q_{f}\right)$ can be made time-dependent through these parameters. Specifically, we adopt here the frozen Gaussian approach, in which the $\gamma_{n}^{(f)}$ is fixed (we also set $\gamma_{n}^{(f)}=\gamma^{(f)} \forall n$ for simplicity), but $q_{n}^{(f)}$ and $p_{n}^{(f)}$ are time-dependent. One can combine these parameters into the complexvalued $z_{n}^{(f)}: 59$

$$
z_{n}^{(f)}=\sqrt{\frac{\gamma_{n}^{(f)}}{2}} q_{n}^{(f)}+\frac{\mathrm{i}}{\hbar} \sqrt{\frac{1}{2 \gamma_{n}^{(f)}}} p_{n}^{(f)}
$$


The time-dependence of the $q_{n}^{(f)}$ and $p_{n}^{(f)}$ parameters (and hence $\left.z_{n}^{(f)}\right)$ leads to the trajectories of the coherent states in the phase space corresponding to the nuclear DoFs $\left\{Q_{f}\right\}$. Among various recipes for equations of motion for $q_{n}^{(f)}$ and $p_{n}^{(f)}$ (or $z_{n}^{(f)}$ ) we choose the Ehrenfest trajectories, which correspond to the variational principle for the wave function including only a single multidimensional coherent state, $\sum_{A} \tilde{a}_{n}^{(A)}(t)\left|\mathbf{z}_{n}(t)\right\rangle|A\rangle:{ }^{43,60}$

$$
\dot{\mathbf{z}}_{n}=-\frac{\mathrm{i}}{\hbar} \frac{\partial H_{n}^{E h r}}{\partial \mathbf{z}_{n}^{*}}
$$

where

$$
H_{n}^{E h r}=\frac{\sum_{A, B} \tilde{a}_{n}^{(A) *} \tilde{a}_{n}^{(B)}\left\langle\mathbf{z}_{n}\left|\hat{H}_{A B}\right| \mathbf{z}_{n}\right\rangle}{\sum_{A} \tilde{a}_{n}^{(A) *} \tilde{a}_{n}^{(A)}}
$$

The $\tilde{a}_{n}^{(A)}(t)$ coefficients obey:

$$
\begin{gathered}
\mathrm{i} \hbar\left(\dot{\tilde{a}}_{n}^{(A)}+\tilde{a}_{n}^{(A)}\left\langle\mathbf{z}_{n} \mid \dot{\mathbf{z}}_{n}\right\rangle\right)=\sum_{B} \tilde{a}_{n}^{(B)}\left\langle\mathbf{z}_{n}\left|\hat{H}_{A B}\right| \mathbf{z}_{n}\right\rangle, \\
A=1, \ldots, \text { number of el. states. }
\end{gathered}
$$

Further, substituting (3) and (2) into the Schrödinger equation (1) and projecting onto $\left\langle\mathbf{z}_{k}(t)\right|\langle A|$ we obtain:

$$
\begin{aligned}
& \mathrm{i} \hbar \sum_{n}\left(\dot{a}_{n}^{(A)}\left\langle\mathbf{z}_{k} \mid \mathbf{z}_{n}\right\rangle+a_{n}^{(A)}\left\langle\mathbf{z}_{k} \mid \dot{\mathbf{z}}_{n}\right\rangle\right)=\sum_{B, n} a_{n}^{(B)}\left\langle\mathbf{z}_{k}\left|\hat{H}_{A B}\right| \mathbf{z}_{n}\right\rangle, \\
& A=1, \ldots, \text { number of el. states; } \quad k=1, \ldots, \text { number of CSs }
\end{aligned}
$$

The coherent states are not orthogonal to each other and thus overlaps $\left\langle\mathbf{z}_{k} \mid \mathbf{z}_{n}\right\rangle$ are retained in the equations. These overlaps can be computed as

$$
\left\langle\mathbf{z}_{k} \mid \mathbf{z}_{n}\right\rangle=\prod_{f}^{\substack{\text { number of } \\ \text { nucl. DoFs }}}\left\langle z_{k}^{(f)} \mid z_{n}^{(f)}\right\rangle
$$

where ${ }^{59,61}$

$$
\left\langle z_{k}^{(f)} \mid z_{n}^{(f)}\right\rangle=\exp \left(z_{k}^{(f) *} z_{n}^{(f)}-\frac{z_{k}^{(f) *} z_{k}^{(f)}}{2}-\frac{z_{n}^{(f) *} z_{n}^{(f)}}{2}\right)
$$


For $k=n$ the overlap is equal to 1 . We note that (13) does not hold if one calculates the overlap of coherent state wave functions with different $\gamma^{(f)}$ parameters.

The solution of (11), i.e., the time-dependent coefficients $a_{n}^{(A)}(t)$ are the last ingredients, complementing the diabatic electronic states and nuclear coherent states, to obtain the molecular wave function. Having these coefficients, one can compute, e.g., the population of the electronic state $|A\rangle$ as:

$$
P^{(A)}=\langle\Psi \mid A\rangle\langle A \mid \Psi\rangle=\sum_{n, k} a_{n}^{(A) *} a_{k}^{(A)}\left\langle\mathbf{z}_{n} \mid \mathbf{z}_{k}\right\rangle
$$

In order to solve (11) we introduce the new coefficients:

$$
c_{k}^{(A)}=\sum_{n} a_{n}^{(A)}\left\langle\mathbf{z}_{k} \mid \mathbf{z}_{n}\right\rangle
$$

Using these new coefficients we can rewrite (11) as follows:

$$
\dot{c}_{k}^{(A)}=-\frac{\mathrm{i}}{\hbar} \sum_{B, n} a_{n}^{(B)}\left\langle\mathbf{z}_{k}\left|\hat{H}_{A B}\right| \mathbf{z}_{n}\right\rangle+\sum_{n} a_{n}^{(A)}\left\langle\dot{\mathbf{z}}_{k} \mid \mathbf{z}_{n}\right\rangle
$$

In (16) (as well as (11)) we encounter overlaps with the timederivative of the coherent states $\left\langle\dot{\mathbf{z}}_{k} \mid \mathbf{z}_{n}\right\rangle$. These overlaps can be computed as

$$
\left\langle\dot{\mathbf{z}}_{k} \mid \mathbf{z}_{n}\right\rangle=\left\langle\mathbf{z}_{k} \mid \mathbf{z}_{n}\right\rangle \sum_{f}\left(\dot{z}_{k}^{(f) *} z_{n}^{(f)}-\operatorname{Re}\left(\dot{z}_{k}^{(f)} z_{k}^{(f) *}\right)\right)
$$

One can obtain this relation (and the overlap formula (13)) starting from the expansion of the coherent state $\left|z_{k}^{(f)}\right\rangle$ in terms of the number states $|n\rangle{ }^{61}$

For more stable numerical integration of coefficients (see (16)) one can rewrite

$$
\begin{aligned}
& a_{n}^{(A)}=b_{n}^{(A)} \exp \left(\frac{\mathrm{i}}{\hbar} S_{n}^{(A)}\right) \\
& c_{k}^{(A)}=d_{k}^{(A)} \exp \left(\frac{\mathrm{i}}{\hbar} S_{k}^{(A)}\right)
\end{aligned}
$$

Where $S_{n}^{(A)}$ is the action obeying: ${ }^{43}$

$$
\dot{S}_{n}^{(A)}=\frac{\mathrm{i} \hbar}{2}\left(\dot{\mathbf{z}}_{n} \mathbf{z}_{n}^{*}-\dot{\mathbf{z}}_{n}^{*} \mathbf{z}_{n}\right)-\left\langle\mathbf{z}_{n}\left|\hat{H}_{A A}\right| \mathbf{z}_{n}\right\rangle
$$


Using the coefficients $b_{n}^{(A)}, d_{k}^{(A)}$, and action $S_{n}^{(A)},(16)$ becomes:

$$
\begin{aligned}
\dot{d}_{k}^{(A)}= & \left\{-\frac{\mathrm{i}}{\hbar}\left[\sum_{n} b_{n}^{(A)} \exp \left(\frac{\mathrm{i}}{\hbar} S_{n}^{(A)}\right)\left\langle\mathbf{z}_{k} \mid \mathbf{z}_{n}\right\rangle \dot{S}_{k}^{(A)}+\sum_{B, n} b_{n}^{(B)} \exp \left(\frac{\mathrm{i}}{\hbar} S_{n}^{(B)}\right)\left\langle\mathbf{z}_{k}\left|\hat{H}_{A B}\right| \mathbf{z}_{n}\right\rangle\right]\right. \\
& \left.+\sum_{n} b_{n}^{(A)} \exp \left(\frac{\mathrm{i}}{\hbar} S_{n}^{(A)}\right)\left\langle\dot{\mathbf{z}}_{k} \mid \mathbf{z}_{n}\right\rangle\right\} \exp \left(-\frac{\mathrm{i}}{\hbar} S_{k}^{(A)}\right)
\end{aligned}
$$

The matrix elements $\left\langle\mathbf{z}_{k}\left|\hat{H}_{A B}\right| \mathbf{z}_{n}\right\rangle$ can be computed analytically for a model Hamiltonian. We used Mathematica ${ }^{62}$ to obtain analytic expressions for the matrix elements.

When a time-independent basis is used (see next subsection), i.e., $\dot{\mathbf{z}}_{n}=0$, then (16) becomes:

$$
\dot{c}_{k}^{(A)}=-\frac{\mathrm{i}}{\hbar} \sum_{B, n} a_{n}^{(B)}\left\langle\mathbf{z}_{k}\left|\hat{H}_{A B}\right| \mathbf{z}_{n}\right\rangle
$$

We note here that the left and the right side of differential equations (20) and (21) contain different coefficients and therefore we use a modified Runge-Kutta fourth order method to numerically integrate (20) and (21) (see Appendix).

\subsection{Sampling initial conditions and basis sets}

We sample initial conditions using the Wigner function ${ }^{63}$ which corresponds to the initial nuclear wave packet $\psi(\mathbf{Q})$ situated on a single electronic state, defined as follows: ${ }^{64}$

$W_{\psi}(\mathbf{Q}, \mathbf{P})=\frac{1}{(2 \pi \hbar)^{d}} \int \exp \left(\mathrm{i} \frac{\mathbf{Q}^{\prime} \mathbf{P}}{\hbar}\right) \psi\left(\mathbf{Q}-\frac{1}{2} \mathbf{Q}^{\prime}\right) \psi^{*}\left(\mathbf{Q}+\frac{1}{2} \mathbf{Q}^{\prime}\right) \mathrm{d} \mathbf{Q}^{\prime}$

Here, $d$ is the number of nuclear DoFs.

Once sampled, $\{\mathbf{Q}, \mathbf{P}\}$ values are combined into $\left\{\mathbf{z}_{n}(t=0)\right\}$, see (7). Due to the fact that coherent states are not orthogonal to each other, problems due to near singular overlap matrix can arise in propagation if a rather large number of the coherent states is sampled. It is thus desired to choose from the sample the set of coherent states which are "not very linear dependent". To solve this issue we apply 
the rank-revealing $\mathrm{QR}$ decomposition to the overlap matrix and select only those states which are associated with the diagonal values of the $\mathrm{R}$ matrix which are larger (in absolute value) than some predefined threshold. To spread initial conditions one can also apply broader distributions, e.g. for a Gaussian wave packet $\psi, W_{\psi}^{1 / 3}(\mathbf{Q}, \mathbf{P})$, $W_{\psi}^{1 / 5}(\mathbf{Q}, \mathbf{P}), W_{\psi}^{1 / 7}(\mathbf{Q}, \mathbf{P})$, etc. can be chosen. This strategy is similar to decreasing the so-called compression parameter in the approach of Shalashilin and Child. ${ }^{65}$ To prepare a fixed basis, however, a more compact distribution (e.g., $\left.W_{\psi}^{5}(\mathbf{Q}, \mathbf{P})\right)$ for sampling initial conditions for trajectories may perform better than the Wigner function itself.

The time-dependent basis is obtained by propagating the selected coherent states with the help of (8), i.e. $\left\{\mathbf{z}_{n}(t)\right\}$ is the basis for the time $t$. We note here that this procedure represents the most straightforward way to generate a moving basis set. More sophisticated techniques have been proposed in literature to improve the moving basis, including coherent state trains and basis function cloning. ${ }^{56}$ The effect of these techniques is not addressed in the present work and is the subject for further investigations.

The time-independent basis is generated by sampling the precomputed trajectories $\left\{\mathbf{z}_{n}(t)\right\}$, as suggested by Saller and Habershon. ${ }^{55}$ Here, we simply select Gaussians every $m$ th time step. The obtained set of CSs is subject to the QR decomposition to avoid problems related to the singularity of the overlap matrix. Such a basis is natively nonlocal and can account for complex spread of the wave packet during propagation in time, as will be shown in the present work. In highly multidimensional systems, with much more than five nuclear DoFs, the generation of the fixed basis in the described manner is most probably prohibitive because of presumably very large number of coherent states needed to obtain converged results. Therefore, the approaches utilizing adaptive time-independent basis sets ${ }^{66-69}$ represent a promising alternative for further exploration.

The initial $a_{n}^{(A)}(0)$ coefficients for the wave function expansion (see (3)) are obtained as a solution of the matrix equation arising through the $\left\langle\mathbf{z}_{k}(0)\right|\langle A|$ projection:

$$
\sum_{n}\left\langle\mathbf{z}_{k}(0) \mid \mathbf{z}_{n}(0)\right\rangle a_{n}^{(A)}(0)=\left\langle\mathbf{z}_{k}(0) \mid \psi^{(A)}(0)\right\rangle
$$

for the initial wave function $|\Psi(0)\rangle=\left|\psi^{(A)}(0)\right\rangle|A\rangle$. 


\subsection{Reference calculations}

To assess the results obtained with the help of the Ehrenfest trajectories of coherent states, we have performed the quantum dynamics calculations on a grid using the second order differences (SODs) method, for the two-dimensional model. For the five-dimensional model the MCTDH calculations of ref. 70 have been used as a reference.

In the SODs method, one propagates the wave packet as:

$$
\psi^{(A)}(\mathbf{Q}, t+\Delta t)=\psi^{(A)}(\mathbf{Q}, t-\Delta t)-2 \frac{\mathrm{i}}{\hbar} \sum_{B} \hat{H}_{A B} \psi^{(B)}(\mathbf{Q}, t) \Delta t
$$

The action of nuclear kinetic energy operator on the wave packet was computed in momentum space. The fast Fourier transform was used to change the representation (from position to momentum, and back). The grid chosen spanned the area of $-3 \leq Q_{1} \leq 10$ and $-5 \leq Q_{2} \leq 5$ with 256 points in each direction. The time step used was 0.001 fs.

\section{Models}

\subsection{Two-state two-dimensional model}

The model for a two-state intersection with two nuclear DoFs $Q_{1}, Q_{2}$ has been adopted from the work of Ferretti et. al. ${ }^{71}$ The Hamiltonian takes on the form

$$
\hat{H}=\hat{H}_{A}|A\rangle\left\langle A\left|+\hat{H}_{B}\right| B\right\rangle\langle B|+\hat{V}| A\rangle\langle B|+\hat{V}| B\rangle\langle A|
$$

with the nuclear operators being

$$
\begin{aligned}
\hat{H}_{A} & =-\frac{\hbar^{2}}{2 m_{1}} \frac{\partial^{2}}{\partial Q_{1}^{2}}-\frac{\hbar^{2}}{2 m_{2}} \frac{\partial^{2}}{\partial Q_{2}^{2}}+\frac{1}{2} k_{x}\left(Q_{1}-X_{1}\right)^{2}+\frac{1}{2} k_{y} Q_{2}^{2} \\
\hat{H}_{B} & =-\frac{\hbar^{2}}{2 m_{1}} \frac{\partial^{2}}{\partial Q_{1}^{2}}-\frac{\hbar^{2}}{2 m_{2}} \frac{\partial^{2}}{\partial Q_{2}^{2}}+\frac{1}{2} k_{x}\left(Q_{1}-X_{2}\right)^{2}+\frac{1}{2} k_{y} Q_{2}^{2}+\Delta \\
\hat{V} & =\Gamma Q_{2} \exp \left(-\alpha\left(Q_{1}-X_{3}\right)^{2}-\beta Q_{2}^{2}\right)
\end{aligned}
$$

The coupling strength is controlled via the $\Gamma$ parameter. $\Gamma=0.01$ corresponds to the weak coupling, whereas $\Gamma=0.08$ is the strong coupling. The other parameters are $X_{1}=4.0, X_{2}=3.0, X_{3}=$ 
$3.0, k_{x}=0.02, k_{y}=0.1, \Delta=0.01, \alpha=3.0, \beta=1.5, m_{1}=$ $20000, m_{2}=6667$. The conical intersection is located at $\left(Q_{1}, Q_{2}\right)=$ $(3.0,0.0)$.

The initial wave packet is a Gaussian, placed on State $A$ and centered at $\left(Q_{1}, Q_{2}\right)=\left(X_{0}, 0\right)$ :

$$
\begin{aligned}
& \psi^{(A)}\left(Q_{1}, Q_{2}, t=0\right)=\frac{1}{\sqrt{\pi \Delta X \Delta Y}} \exp \left(-\frac{1}{2} \frac{\left(Q_{1}-X_{0}\right)^{2}}{(\Delta X)^{2}}-\frac{1}{2} \frac{Q_{2}^{2}}{(\Delta Y)^{2}}\right) \\
& \psi^{(B)}\left(Q_{1}, Q_{2}, t=0\right)=0
\end{aligned}
$$

Here, $\Delta X=0.150$ and $\Delta Y=0.197$.

Calculating the Wigner function of $\psi^{(A)}$ yields multivariate normal distribution with the following mean and covariance matrix:

$$
\begin{aligned}
& \text { mean }=\left(\begin{array}{cccc}
\left(X_{0}\right. & 0 & 0 & 0
\end{array}\right) \\
& \operatorname{cov}=\left(\begin{array}{cccc}
\frac{(\Delta X)^{2}}{2} & 0 & 0 & 0 \\
0 & \frac{(\Delta Y)^{2}}{2} & 0 & 0 \\
0 & 0 & \frac{\hbar^{2}}{2(\Delta X)^{2}} & 0 \\
0 & 0 & 0 & \frac{\hbar^{2}}{2(\Delta Y)^{2}}
\end{array}\right)
\end{aligned}
$$

To select more states, which do not overlap too much, we used $3 \times$ cov, $5 \times \operatorname{cov}$ or $7 \times \operatorname{cov}$ as the covariance matrix. These choices correspond to the $W_{\psi}^{1 / 3}(\mathbf{Q}, \mathbf{P}), W_{\psi}^{1 / 5}(\mathbf{Q}, \mathbf{P})$, and $W_{\psi}^{1 / 7}(\mathbf{Q}, \mathbf{P})$ functions, respectively.

\subsection{Three-state five-dimensional model}

The model for a three-state intersection with five nuclear DoFs has been adopted from the work of Krause and Matsika. ${ }^{70}$ The Hamiltonian takes on the form

$$
\begin{aligned}
\hat{H} & =\hat{H}_{A}|A\rangle\left\langle A\left|+\hat{H}_{B}\right| B\right\rangle\left\langle B\left|+\hat{H}_{C}\right| C\right\rangle\langle C| \\
& +\hat{V}_{A B}|A\rangle\left\langle B\left|+\hat{V}_{A B}\right| B\right\rangle\left\langle A\left|+\hat{V}_{A C}\right| A\right\rangle\left\langle C\left|+\hat{V}_{A C}\right| C\right\rangle\left\langle A\left|+\hat{V}_{B C}\right| B\right\rangle\left\langle C\left|+\hat{V}_{B C}\right| C\right\rangle\langle B|
\end{aligned}
$$


with the nuclear operators being

$$
\begin{aligned}
\hat{H}_{A}= & -\frac{\omega_{e}}{2} \frac{\partial^{2}}{\partial Q_{1}^{2}}-\frac{\omega_{e}}{2} \frac{\partial^{2}}{\partial Q_{2}^{2}}-\frac{\omega_{t}}{2} \frac{\partial^{2}}{\partial Q_{3}^{2}}-\frac{\omega_{t}}{2} \frac{\partial^{2}}{\partial Q_{4}^{2}}-\frac{\omega_{t}}{2} \frac{\partial^{2}}{\partial Q_{5}^{2}} \\
& +\frac{\omega_{e}}{2} Q_{1}^{2}+\frac{\omega_{e}}{2} Q_{2}^{2}+\frac{\omega_{t}}{2} Q_{3}^{2}+\frac{\omega_{t}}{2} Q_{4}^{2}+\frac{\omega_{t}}{2} Q_{5}^{2} \\
& +F_{e} \frac{1}{2} Q_{1}-F_{e} \frac{\sqrt{3}}{2} Q_{2} \\
\hat{H}_{B}= & -\frac{\omega_{e}}{2} \frac{\partial^{2}}{\partial Q_{1}^{2}}-\frac{\omega_{e}}{2} \frac{\partial^{2}}{\partial Q_{2}^{2}}-\frac{\omega_{t}}{2} \frac{\partial^{2}}{\partial Q_{3}^{2}}-\frac{\omega_{t}}{2} \frac{\partial^{2}}{\partial Q_{4}^{2}}-\frac{\omega_{t}}{2} \frac{\partial^{2}}{\partial Q_{5}^{2}} \\
& +\frac{\omega_{e}}{2} Q_{1}^{2}+\frac{\omega_{e}}{2} Q_{2}^{2}+\frac{\omega_{t}}{2} Q_{3}^{2}+\frac{\omega_{t}}{2} Q_{4}^{2}+\frac{\omega_{t}}{2} Q_{5}^{2} \\
& +F_{e} \frac{1}{2} Q_{1}+F_{e} \frac{\sqrt{3}}{2} Q_{2} \\
\hat{H}_{C}= & -\frac{\omega_{e}}{2} \frac{\partial^{2}}{\partial Q_{1}^{2}}-\frac{\omega_{e}}{2} \frac{\partial^{2}}{\partial Q_{2}^{2}}-\frac{\omega_{t}}{2} \frac{\partial^{2}}{\partial Q_{3}^{2}}-\frac{\omega_{t}}{2} \frac{\partial^{2}}{\partial Q_{4}^{2}}-\frac{\omega_{t}}{2} \frac{\partial^{2}}{\partial Q_{5}^{2}} \\
& +\frac{\omega_{e}}{2} Q_{1}^{2}+\frac{\omega_{e}}{2} Q_{2}^{2}+\frac{\omega_{t}}{2} Q_{3}^{2}+\frac{\omega_{t}}{2} Q_{4}^{2}+\frac{\omega_{t}}{2} Q_{5}^{2} \\
& -F_{e} Q_{1} \\
\hat{V}_{A B}= & -F_{t} Q_{4} \\
\hat{V}_{A C}= & -F_{t} Q_{3} \\
\hat{V}_{B C}= & -F_{t} Q_{5}
\end{aligned}
$$

The parameters used are $\omega_{e}=\omega_{t}=0.0036749324, F_{e}=0.0036749324$, $F_{t}=2 F_{e}$. The intersection of three PESs is located at $\left(Q_{1}, Q_{2}, Q_{3}, Q_{4}, Q_{5}\right)=$ $(0,0,0,0,0)$.

The initial wave packet is a Gaussian, placed on State $C$ and centered at $\left(Q_{1}, Q_{2}, Q_{3}, Q_{4}, Q_{5}\right)=(0,0,0,0,0)$ (i.e. directly at the intersection):

$$
\begin{aligned}
& \psi^{(A)}\left(Q_{1}, Q_{2}, Q_{3}, Q_{4}, Q_{5}, t=0\right)=0 \\
& \psi^{(B)}\left(Q_{1}, Q_{2}, Q_{3}, Q_{4}, Q_{5}, t=0\right)=0 \\
& \psi^{(C)}\left(Q_{1}, Q_{2}, Q_{3}, Q_{4}, Q_{5}, t=0\right)=\prod_{f=1}^{5}\left\{\left(\frac{1}{\pi}\right)^{\frac{1}{4}} \exp \left(-\frac{1}{2} Q_{f}^{2}\right)\right\}
\end{aligned}
$$

The Wigner function of $\psi^{(C)}$ is the multivariate normal distribution 
with the following mean and covariance matrix:

$$
\begin{aligned}
& \operatorname{mean}=\left(\begin{array}{ccccccccccc}
0 & 0 & 0 & 0 & 0 & 0 & 0 & 0 & 0 & 0
\end{array}\right) \\
& \operatorname{cov}=\left(\begin{array}{ccccccccccc}
\frac{1}{2} & 0 & 0 & 0 & 0 & 0 & 0 & 0 & 0 & 0 \\
0 & \frac{1}{2} & 0 & 0 & 0 & 0 & 0 & 0 & 0 & 0 \\
0 & 0 & \frac{1}{2} & 0 & 0 & 0 & 0 & 0 & 0 & 0 \\
0 & 0 & 0 & \frac{1}{2} & 0 & 0 & 0 & 0 & 0 & 0 \\
0 & 0 & 0 & 0 & \frac{1}{2} & 0 & 0 & 0 & 0 & 0 \\
0 & 0 & 0 & 0 & 0 & \frac{\hbar^{2}}{2} & 0 & 0 & 0 & 0 \\
0 & 0 & 0 & 0 & 0 & 0 & \frac{\hbar^{2}}{2} & 0 & 0 & 0 \\
0 & 0 & 0 & 0 & 0 & 0 & 0 & \frac{\hbar^{2}}{2} & 0 & 0 \\
0 & 0 & 0 & 0 & 0 & 0 & 0 & 0 & \frac{\hbar^{2}}{2} & 0 \\
0 & 0 & 0 & 0 & 0 & 0 & 0 & 0 & 0 & \frac{\hbar^{2}}{2}
\end{array}\right)
\end{aligned}
$$

We have also used $\frac{1}{5} \times \operatorname{cov}$ as a covariance matrix in the case of a fixed basis, since with it we were able to obtain better agreement than with cov.

\section{Results and Discussion}

\subsection{Two-state two-dimensional model}

For the two-state two-dimensional intersection model, we study two cases: (i) the initial wave packet is centered at $\left(Q_{1}, Q_{2}\right)=(2.0,0.0)$ and placed on state $\mathrm{A}$, and (ii) the initial wave packet is centered at $\left(Q_{1}, Q_{2}\right)=(5.2,0.0)$ and placed again on state $\mathrm{A}$. These two cases were considered previously in literature, mainly to asses the performance of various methods for nonadiabatic dynamics. ${ }^{47,50,71-73}$ The cross sections of the diabatic potentials along with the initial conditions and the energy of the conical intersection are shown in Fig. 1. 


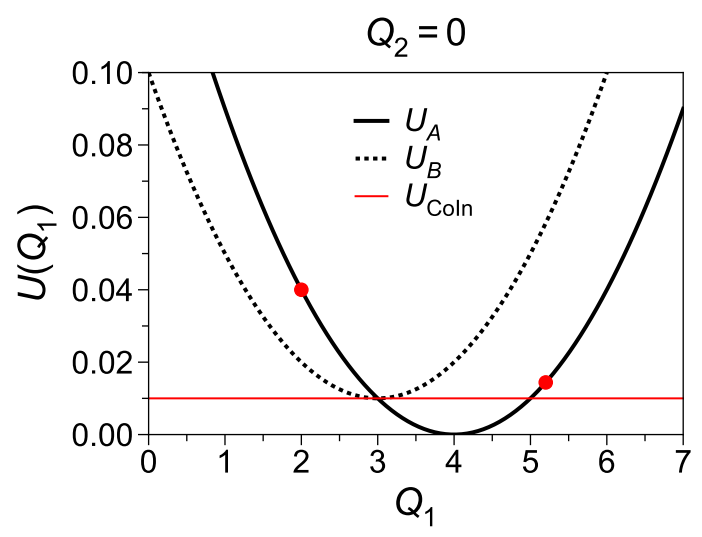

Figure 1: Diabatic potentials for states $A$ and $B$ as a function of $Q_{1}$ at fixed $Q_{2}=0$. Red dots indicate two considered initial positions of the wave packet, placed on state $A$. Red thin line marks the energy of the conical intersection (CoIn).

For both cases the wave packet momentum is initially zero. When starting at $\left(Q_{1}, Q_{2}\right)=(2.0,0.0)$, from the left of the conical intersection, the excess energy, $U_{\text {initial }}-U_{\text {CoIn }}$, is $\sim 7$ times larger than when starting at $\left(Q_{1}, Q_{2}\right)=(5.2,0.0)$, from the right of the conical intersection. Namely, for case (i) the excess energy is 0.03 , and for case (ii) the excess energy is about 0.0044 . It should be noted that the second case is particularly difficult for conventional surface hopping approach, as discussed by Yang et al. ${ }^{72}$ In this subsection, we will discuss how the CSs approaches utilizing moving and fixed basis sets perform for the different initial conditions described above. For each initial condition we will consider scenarios of weak $(\Gamma=0.01)$ and strong $(\Gamma=0.08)$ couplings (see eq. (26c)). In all cases considered below for the two-state intersection we set $\gamma^{(1)}=44.44$ and $\gamma^{(2)}=25.7$, what corresponds to the width of the initial wave packet.

\subsubsection{Initial position $\left(Q_{1}, Q_{2}\right)=(2.0,0.0)$}

For weak coupling $(\Gamma=0.01)$, the diabatic state populations for the model conical intersection of two states obtained with the moving and fixed basis sets are shown in Fig. 2 (top panels). 

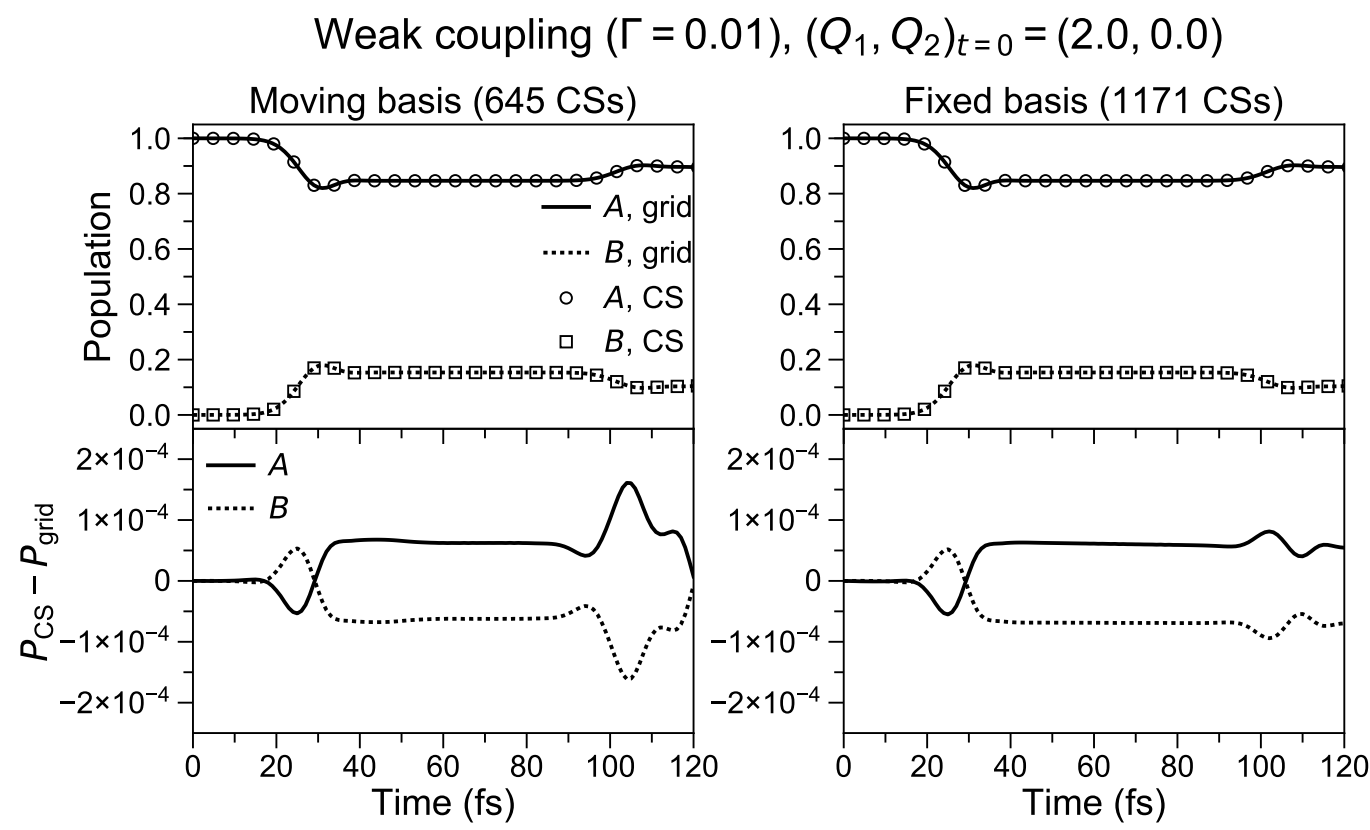

Figure 2: Diabatic populations for weak coupling $(\Gamma=0.01)$ obtained with the moving and fixed CS basis sets, compared to propagation on a grid, for the initial wave packet centered at $\left(Q_{1}, Q_{2}\right)=(2.0,0.0)$ and located on state $A$. The differences between coherent state and grid populations, $P_{\mathrm{CS}}-P_{\text {grid }}$, are also shown.

As can be seen (particularly from the difference graphs, $P_{\mathrm{CS}}-P_{\text {grid }}$, in the bottom panels of Fig. 2), the excellent agreement between the CS method and propagation on a grid is achieved. We note that the large number of Ehrenfest trajectories, namely 645, is used in the case of the moving basis. These 645 initial coherent states were chosen by means of QR decomposition of the overlap matrix of 15,000 coherent states sampled from the $W_{\psi}^{1 / 3}(\mathbf{Q}, \mathbf{P})$ distribution, retaining only states corresponding to the diagonal values of the $\mathrm{R}$ matrix, whose moduli are larger than $10^{-10}$. For a fixed basis set, the number of basis functions is even larger, 1171. However, they are fixed and are sampled from 100 trajectories. The initial conditions for these 100 trajectories were merely sampled from the Wigner distribution, i.e. $W(\mathbf{Q}, \mathbf{P})$. Then, the Gaussians were sampled from these trajectories with a period of $100 \mathrm{au}(2.419 \mathrm{fs})$. The whole set of chosen fixed functions was then subject to the QR decomposition to generate the final fixed basis set, using the threshold of $10^{-10}$. For all cases considered in this work, we 
used the time step of 1 au to propagate the moving basis and the time step of $10 \mathrm{au}$ to propagate parent trajectories for the fixed basis. The time step for propagation of coefficients was 2 au for the both basis sets.

Here and in what follows, the numbers of basis functions used for propagation were chosen after many tests and correspond to the best results (in terms of reproducing reference time-dependent populations) out of the pool of many trials. We note that the seemingly obvious strategy of simply enlarging the number of basis functions will fail due to singularities of the overlap matrix. Moreover, the QR decomposition done in the beginning does not guarantee the "smooth" propagation afterwards. Here, to overcome the possible issues, one can apply the strategies used for generation of adaptive basis sets ${ }^{67}$, but this will not be addressed in the present work. Quantitatively, the difference in populations between the CS methods and the propagation on the grid is $\sim 10^{-4}$ for the weak coupling, with the used bases.

For strong coupling $(\Gamma=0.08)$, the populations are also in very good agreement with those obtained by the reference calculations (propagation on a grid), see Fig. 3. 


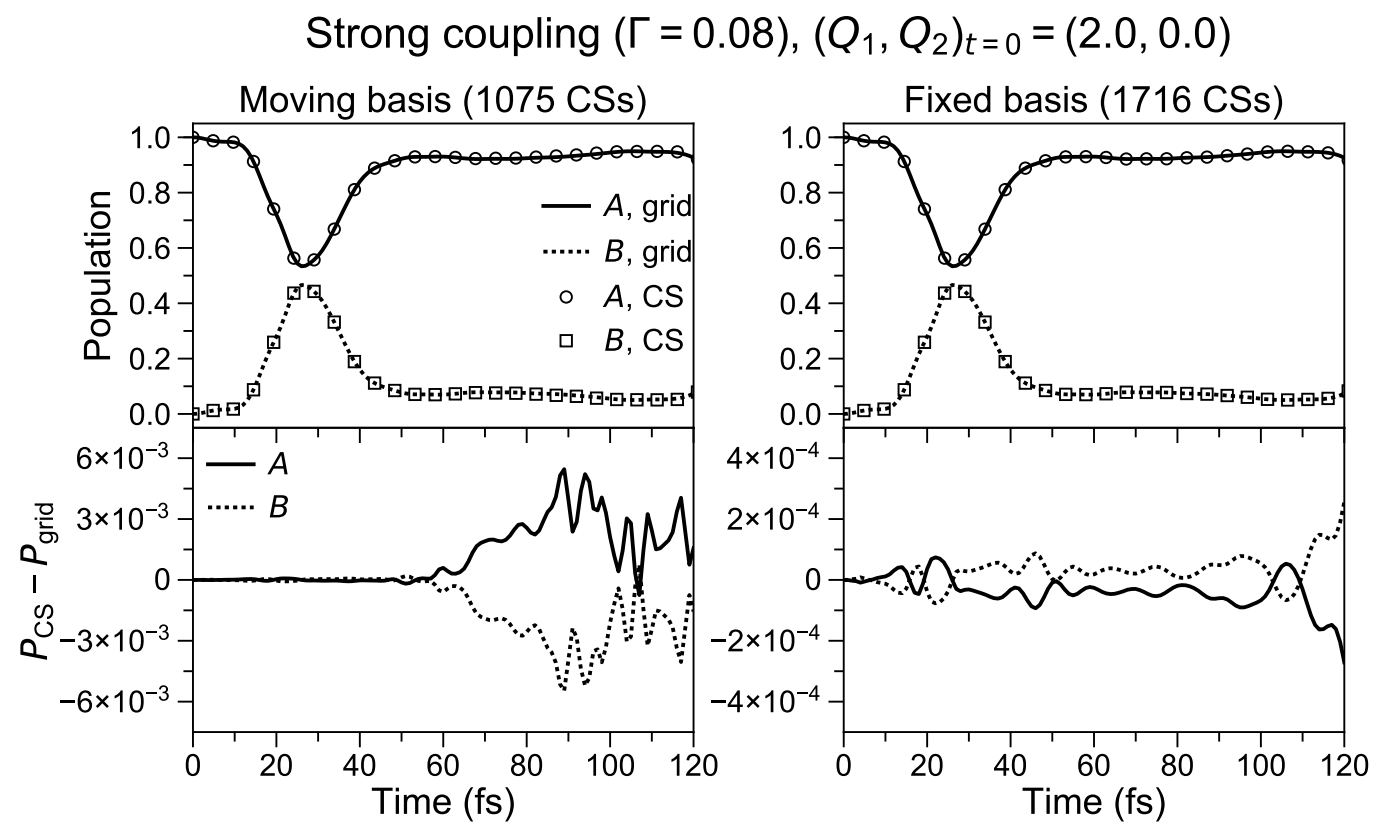

Figure 3: Diabatic populations for strong coupling $(\Gamma=0.08)$ obtained with the moving and fixed CS basis sets, compared to propagation on a grid, for the initial wave packet centered at $\left(Q_{1}, Q_{2}\right)=(2.0,0.0)$ and located on state $A$. The differences between coherent state and grid populations, $P_{\mathrm{CS}}-P_{\text {grid }}$, are also shown.

Quantitatively, the difference in populations between the CS methods and the propagation on the grid for the strong coupling is $\sim 10^{-3}$ with the used moving basis and $\sim 10^{-4}$ with the fixed one. The number of basis CS functions used for the strong coupling case is 1075 (moving basis) and 1716 (fixed basis), i.e. higher than the corresponding numbers for the example of weak coupling described above. Again, we notice that 1716 fixed basis functions were selected from 100 Ehrenfest trajectories, by applying the same procedure as for the weak coupling (with the threshold of $10^{-10}$ ). The initial conditions for 1075 moving basis coherent states, in turn, were selected by using the QR decomposition of the overlap matrix of 20,000 CSs sampled from $W_{\psi}^{1 / 5}(\mathbf{Q}, \mathbf{P})$, with subsequent filtering with the $10^{-10}$ threshold.

The wave packet evolution is also very well captured by both CS approaches, i.e. with (i) moving and (ii) fixed basis sets, for both coupling cases (see Figs. S1 and S2 for details). However, there are certain differences which become obvious upon detailed comparison of 
the results obtained with different methods.

For weak coupling, we compare the wave packets at the end of propagation, at 120 fs (Fig. 4). To better unveil the main features of the wave packet we exclude from analysis all values which are smaller than a certain threshold. Practically, we set white color in the colormap for all such values. It is seen that the wave packet associated with state $B$ looks slightly different when comparing the moving basis with the fixed one. Namely, the parts located around the CoIn (the latter is centered at $\left.\left(Q_{1}, Q_{2}\right)=(3.0,0.0)\right)$ differ in shape of boundaries. Overall, however, the main features of the nuclear wave function at $120 \mathrm{fs}$ are well reproduced with the both, moving and fixed, basis sets. The $\left|\psi^{(A)}(\mathbf{Q}, t)\right|^{2}$ components calculated with both CS approaches resemble the grid reference equally well.

For strong coupling, more complex patterns emerge upon wave packet evolution. The overall agreement with the reference calculations is very good (see Fig. S2 for details). Not surprisingly, however, some deviations from the reference are observed. First, for times of 70-90 fs the wave packet associated with state $B$ exhibits the "tail" located near $Q_{1} \approx 3$, as follows from the reference calculations. This "tail" clearly arises when the fixed basis set is used within the CS method, but, contrarily, this feature is absent in the case of the moving basis. The detailed comparison for time of $70 \mathrm{fs}$ is presented in Fig. 5, where the absence of the "tail" is clearly identifiable in the upper right panel (state $B$, moving basis). Thus, the fixed basis captures better the nonlocality emerging during the wave packet evolution, for the given studied case. Moreover, for still larger times, $\gtrsim 100 \mathrm{fs}$, the wave packet obtained with the fixed basis is closer to the grid propagation reference, than the corresponding wave packet built from the moving CS functions (see Fig. 6 for times of 110 and 120 fs). Importantly, both CS approaches lead to symmetry breaking, with respect to the plane $Q_{2}=0$, whereas the symmetry is preserved in the reference propagation. One could use symmetric initial conditions to overcome this issue, though such a sampling would be specific to the given problem rather than generally applicable. 


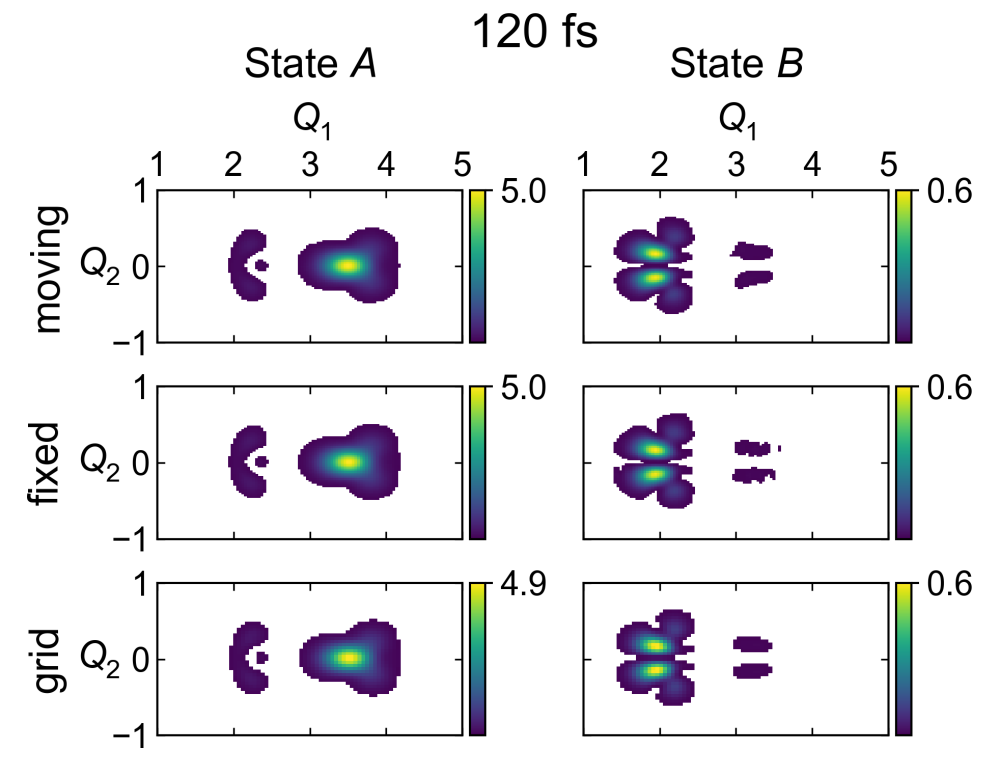

Figure 4: Diabatic wave packet $\left(\left|\psi^{(A)}(\mathbf{Q}, t)\right|^{2}\right.$ and $\left.\left|\psi^{(B)}(\mathbf{Q}, t)\right|^{2}\right)$ at 120 fs for the case of weak coupling $(\Gamma=0.01)$ and the initial position $\left(Q_{1}, Q_{2}\right)=$ $(2.0,0.0)$. Colorbars span the range from zero to the maximum for a given state at a current time. These maxima are shown as tick labels of colorbars (rounded to a 1st decimal place). Values $\left|\psi^{(A)}(\mathbf{Q}, t)\right|^{2}<5 \times 10^{-2}$ and $\left|\psi^{(B)}(\mathbf{Q}, t)\right|^{2}<5 \times 10^{-3}$ are represented in white to highlight the differences in obtained results. 


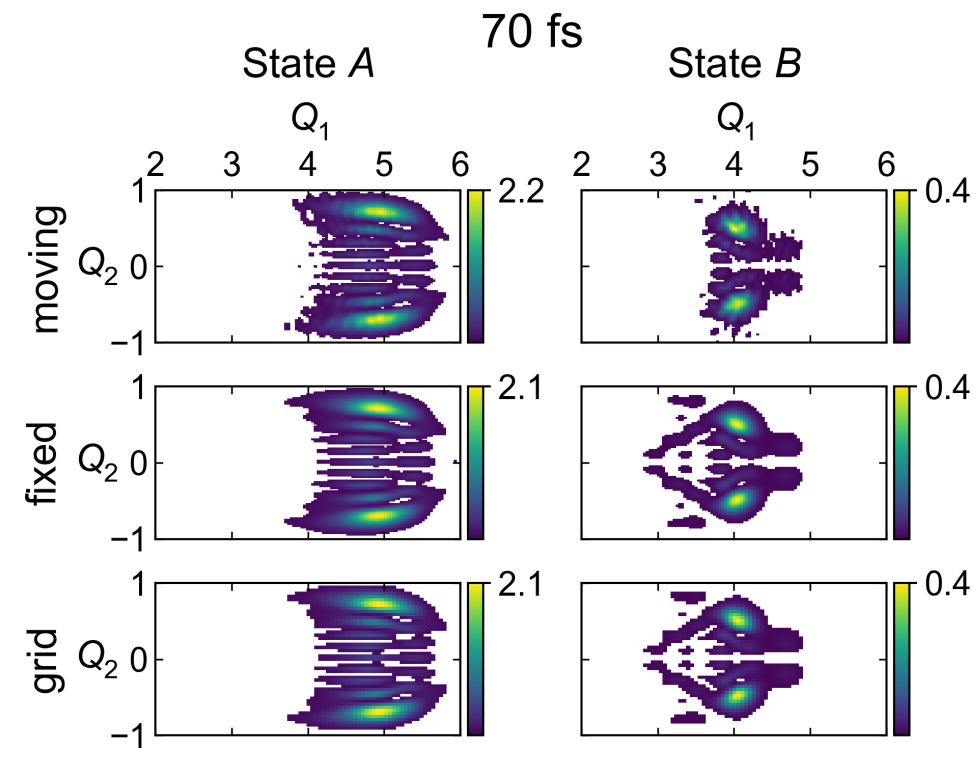

Figure 5: Diabatic wave packet $\left(\left|\psi^{(A)}(\mathbf{Q}, t)\right|^{2}\right.$ and $\left.\left|\psi^{(B)}(\mathbf{Q}, t)\right|^{2}\right)$ at 70 fs for the case of strong coupling $(\Gamma=0.08)$ and the initial position $\left(Q_{1}, Q_{2}\right)=$ $(2.0,0.0)$. Colorbars span the range from zero to the maximum for a given state at a current time. These maxima are shown as tick labels of colorbars (rounded to a 1st decimal place). Values $\left|\psi^{(A)}(\mathbf{Q}, t)\right|^{2}<5 \times 10^{-2}$ and $\left|\psi^{(B)}(\mathbf{Q}, t)\right|^{2}<5 \times 10^{-3}$ are represented in white to highlight the differences in obtained results. 


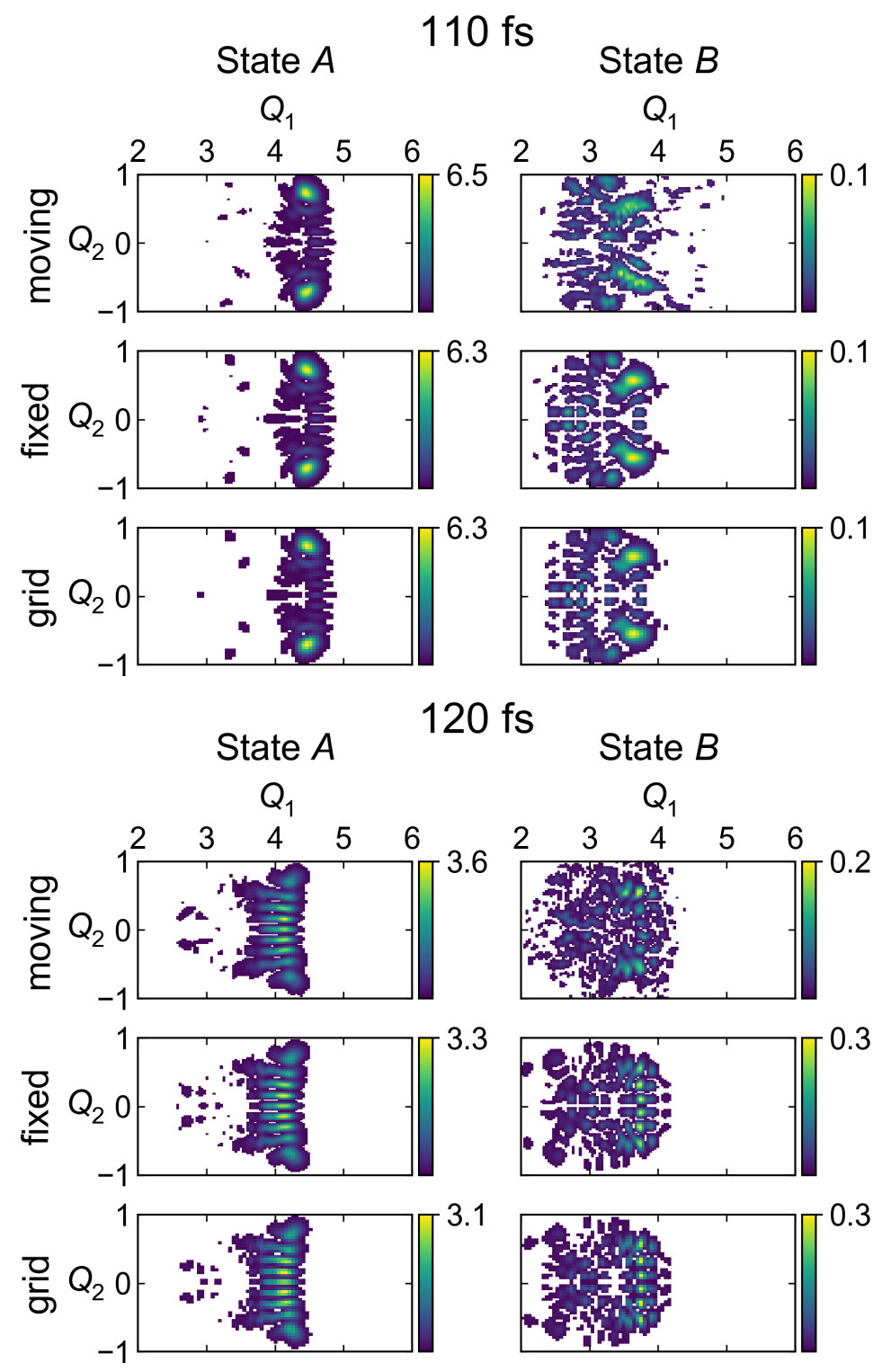

Figure 6: Diabatic wave packet $\left(\left|\psi^{(A)}(\mathbf{Q}, t)\right|^{2}\right.$ and $\left.\left|\psi^{(B)}(\mathbf{Q}, t)\right|^{2}\right)$ at 110 fs (top) and 120 fs (bottom) for the case of strong coupling $(\Gamma=0.08)$ and the initial position $\left(Q_{1}, Q_{2}\right)=(2.0,0.0)$. Colorbars span the range from zero to the maximum for a given state at a current time. These maxima are shown as tick labels of colorbars (rounded to a 1st decimal place). Values $\left|\psi^{(A)}(\mathbf{Q}, t)\right|^{2}<5 \times 10^{-2}$ and $\left|\psi^{(B)}(\mathbf{Q}, t)\right|^{2}<5 \times 10^{-3}$ are represented in white to highlight the differences in obtained results. 


\subsubsection{Initial position $\left(Q_{1}, Q_{2}\right)=(5.2,0.0)$}

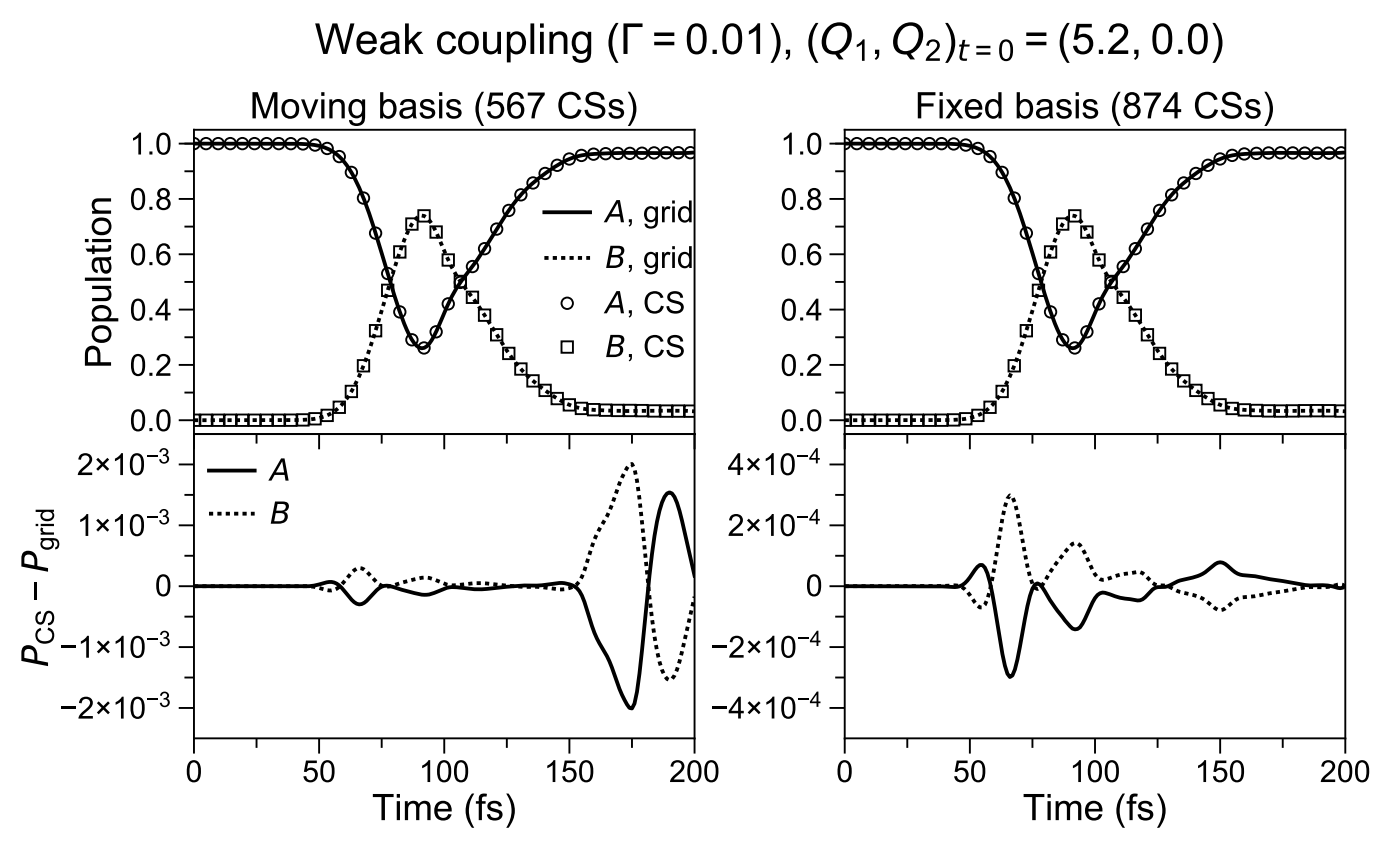

Figure 7: Diabatic populations for weak coupling $(\Gamma=0.01)$ obtained with the moving and fixed CS basis sets, compared to propagation on a grid, for the initial wave packet centered at $\left(Q_{1}, Q_{2}\right)=(5.2,0.0)$ and located on state $A$. The differences between coherent state and grid populations, $P_{\mathrm{CS}}-P_{\text {grid }}$, are also shown.

In this subsection we demonstrate how the CS methods perform when the wave packet is initially placed at $\left(Q_{1}, Q_{2}\right)=(5.2,0.0)$, again on state $A$. This case is difficult for surface hopping methods, since the initial energy is only a little bit higher than the energy of the conical intersection (see Fig. 1).

The diabatic populations in the case of weak coupling $(\Gamma=0.01)$ are presented in Fig. 7. Again, excellent agreement between the CS method and the reference calculations is observed. We note that the accuracy of the populations obtained with the CS method and presented in Fig. 7 is better than the accuracy of the results reported for this case so far, ${ }^{50,72}$ specifically for the larger times region. In our simulations, 567 and 874 CSs were used for the moving and fixed basis strategies, respectively. The $567 \mathrm{CSs}$ in the case of time-dependent 
basis were chosen using the QR decomposition of the overlap matrix of the 15,000 CSs sampled from $W_{\psi}^{1 / 3}(\mathbf{Q}, \mathbf{P})$. The used threshold for retaining the states was $5 \times 10^{-9}$. For the fixed basis, again 100 trajectories with initial conditions sampled form $W(\mathbf{Q}, \mathbf{P})$ were used to sample time-independent functions every $100 \mathrm{au}$. The sampled set was subject to the QR decomposition and the threshold of $10^{-10}$. The population differences between the CS method and reference propagation on a grid are on the order of $10^{-3}$ with the moving basis, and $10^{-4}$ with the fixed basis.

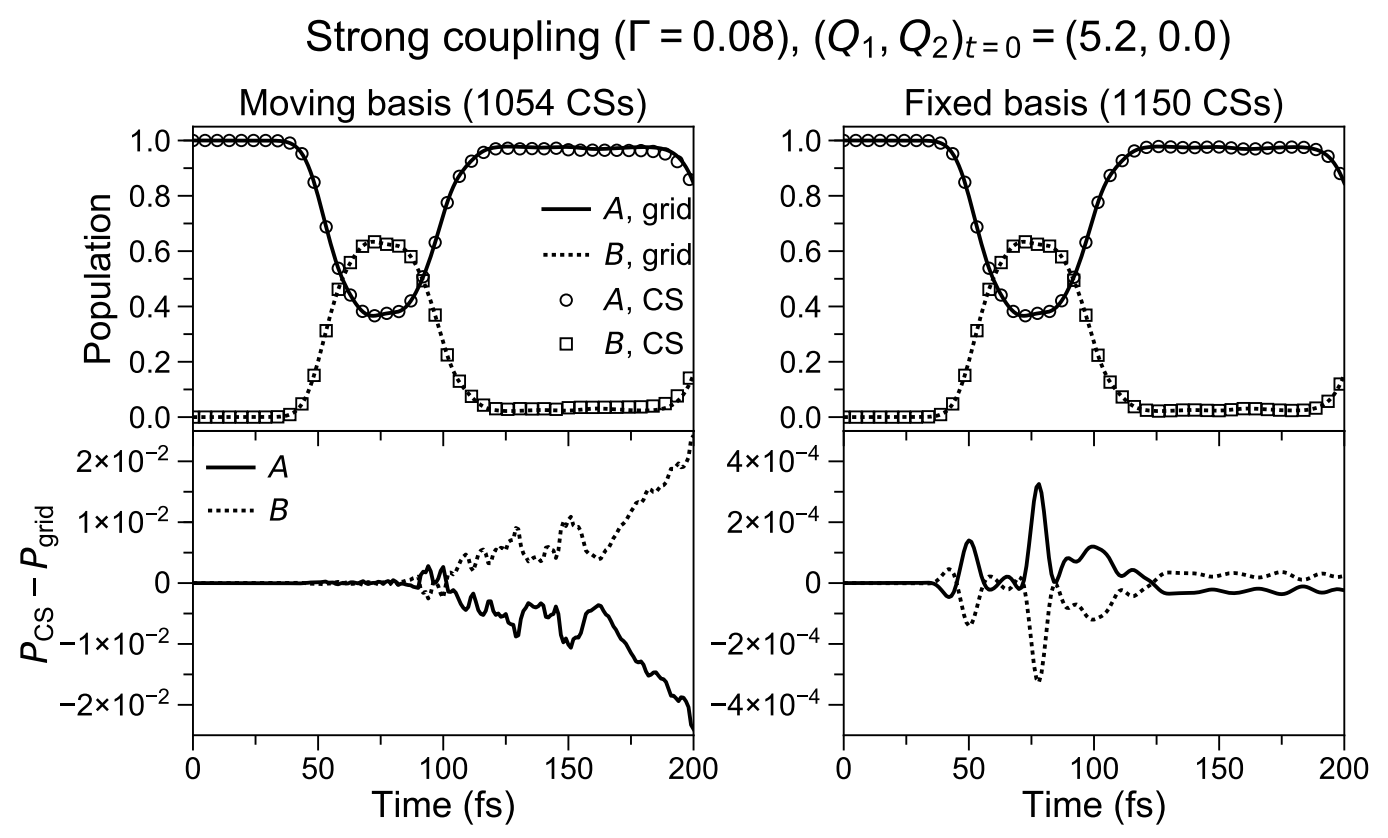

Figure 8: Diabatic populations for strong coupling $(\Gamma=0.08)$ obtained with the moving and fixed CS basis sets, compared to propagation on a grid, for the initial wave packet centered at $\left(Q_{1}, Q_{2}\right)=(5.2,0.0)$ and located on state $A$. The differences between coherent state and grid populations, $P_{\mathrm{CS}}-P_{\text {grid }}$, are also shown.

The case of the strong coupling $(\Gamma=0.08)$ is also well described by the CS method (Fig. 8). Here, though, the fixed basis performs distinctly better than the moving one. 1150 (fixed basis) vs. 1054 (moving basis) functions were used. The $1054 \mathrm{CSs}$ for the moving basis are the outcome of the QR decomposition of $20,000 \times 20,000$ overlap matrix of 20,000 CSs sampled from the $W_{\psi}^{1 / 7}(\mathbf{Q}, \mathbf{P})$ distribution, after excluding 
the states corresponding to the threshold of $5 \times 10^{-6}$. Here, we used the broad $W_{\psi}^{1 / 7}(\mathbf{Q}, \mathbf{P})$ distribution, since the attempts with narrower distributions were less successful, either resulting in less accurate results or in the "explosion" of propagation due to arising linear dependencies. The 1150 fixed basis functions were generated in the same way as described in the previous paragraph for the weak coupling case (using the threshold of $10^{-10}$ ). Quantitatively, the population differences are on the order of $10^{-2}$ with the moving basis set and $10^{-4}$ with the fixed one.

The wave packet evolutions are overall well reproduced for both coupling strengths and both types of basis set, with the exception of the long time dynamics with the moving basis for the strong coupling (see Figs. S3 and S4 for details).

For weak coupling, the detailed comparison of the wave packets at the end of the simulation ( $t=200 \mathrm{fs}$ ) reveals that the fixed basis performs better than the moving basis in reproducing the reference wave packet appearance (Fig. 9).

For strong coupling, as already mentioned, the fixed basis performs much better than the moving basis used. So, the wave packet associated with state $B$ is virtually destroyed at $180 \mathrm{fs}$, when the moving basis is used (Fig. 10, top panel). On the contrary, the fixed basis set performs remarkably well. At 200 fs the dominant feature (corresponding to the maximal values) of $\left|\psi^{(B)}(\mathbf{Q}, t)\right|^{2}$ is somewhat restored, in the case of the moving basis set, apparently due to the population transfer from state $A$ (Fig. 10, bottom panel). We note that the main features of the wave packet associated with state $A$ are well captured also with the moving basis. This state is almost exclusively populated in the long time dynamics. Thus, the described deviations in the wave packet associated with state $B$ do not lead to dramatic changes in the populations (see Fig. 8, left panel). Interestingly, for this case we were able to better reproduce the reference dynamics (in comparison to the results obtained with the given moving basis) with a fixed basis of a smaller size than the size of the given moving basis (namely 880 fixed CSs, not shown). Of course, the comparison of basis sets simply by means of comparison of the numbers of basis functions is oversimplified, since it is possible to have a situation when initial moving basis functions are spread out very much in the nuclear phase space, thus leading to many unnecessary traveling functions in the course of propagation. 


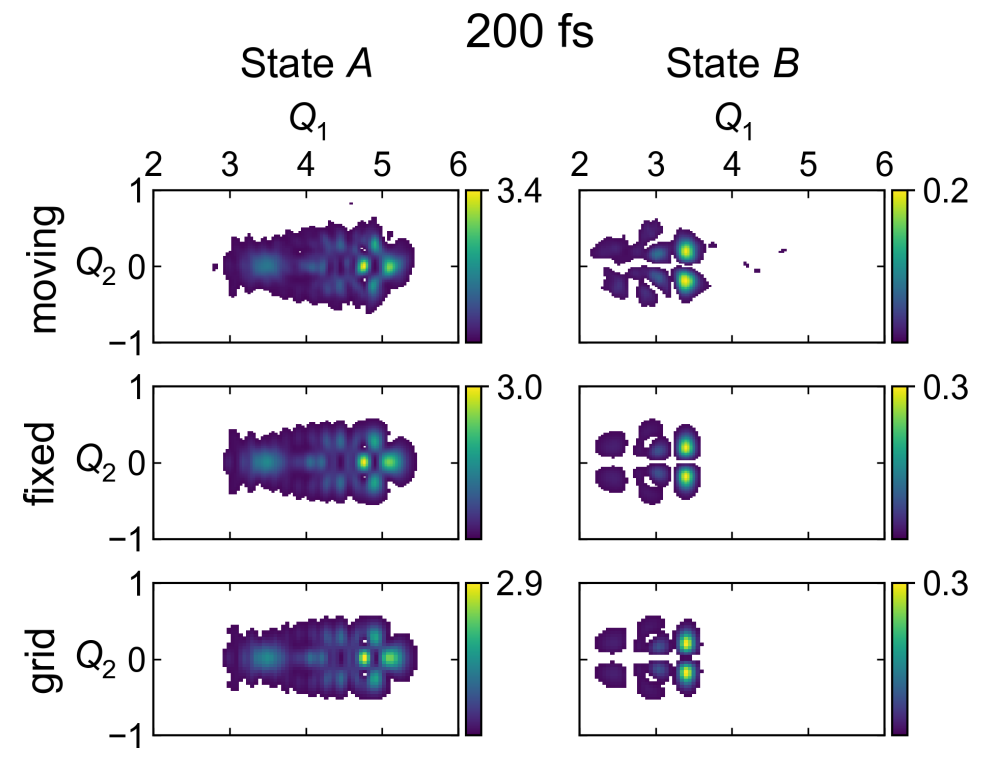

Figure 9: Diabatic wave packet $\left(\left|\psi^{(A)}(\mathbf{Q}, t)\right|^{2}\right.$ and $\left.\left|\psi^{(B)}(\mathbf{Q}, t)\right|^{2}\right)$ at 200 fs for the case of weak coupling $(\Gamma=0.01)$ and the initial position $\left(Q_{1}, Q_{2}\right)=$ $(5.2,0.0)$. Colorbars span the range from zero to the maximum for a given state at a current time. These maxima are shown as tick labels of colorbars (rounded to a 1st decimal place). Values $\left|\psi^{(A)}(\mathbf{Q}, t)\right|^{2}<5 \times 10^{-2}$ and $\left|\psi^{(B)}(\mathbf{Q}, t)\right|^{2}<5 \times 10^{-3}$ are represented in white to highlight the differences in obtained results. 


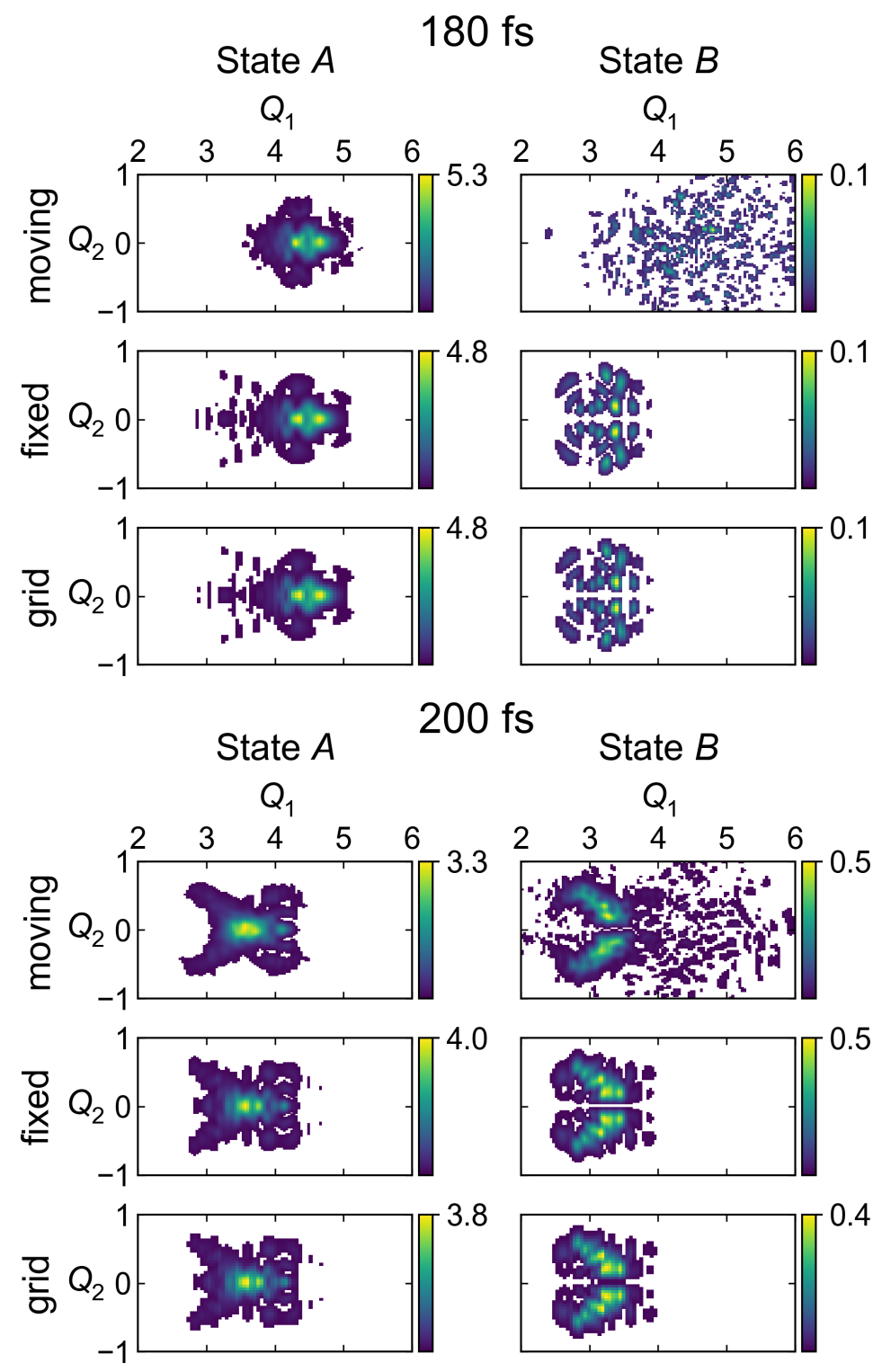

Figure 10: Diabatic wave packet $\left(\left|\psi^{(A)}(\mathbf{Q}, t)\right|^{2}\right.$ and $\left.\left|\psi^{(B)}(\mathbf{Q}, t)\right|^{2}\right)$ at 180 fs and $200 \mathrm{fs}$ for the case of strong coupling $(\Gamma=0.08)$ and the initial position $\left(Q_{1}, Q_{2}\right)=(5.2,0.0)$. Colorbars span the range from zero to the maximum for a given state at a current time. These maxima are shown as tick labels of colorbars (rounded to a 1st decimal place). Values $\left|\psi^{(A)}(\mathbf{Q}, t)\right|^{2}<5 \times 10^{-2}$ and $\left|\psi^{(B)}(\mathbf{Q}, t)\right|^{2}<5 \times 10^{-3}$ are represented in white to highlight the differences in obtained results. 


\subsection{Three-state five-dimensional model}
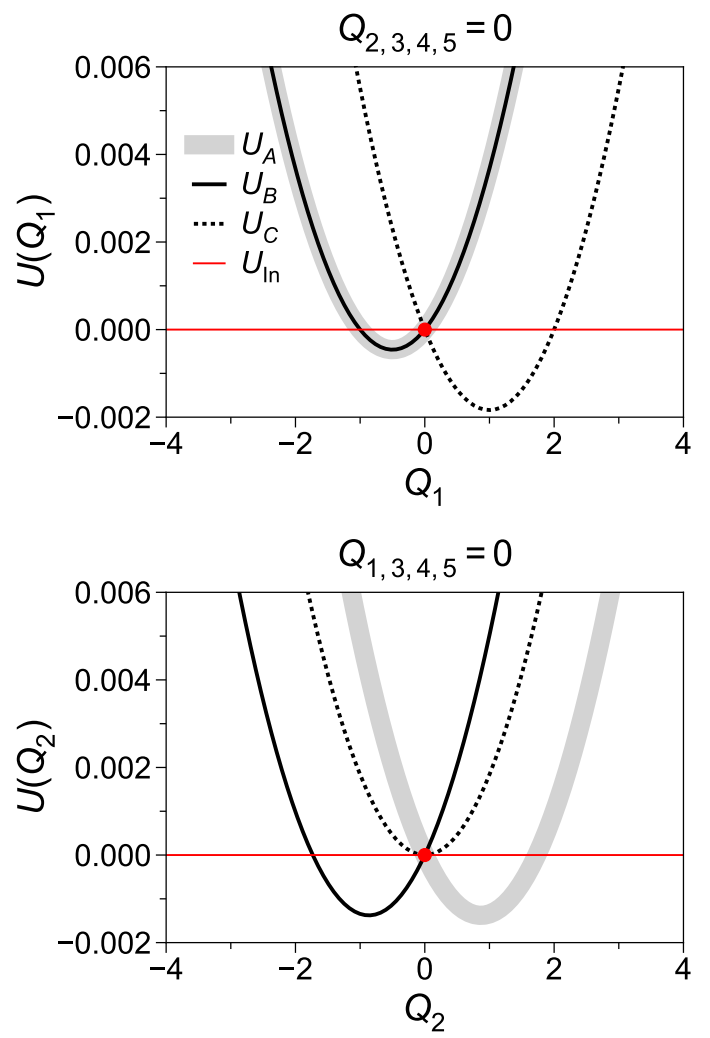

Figure 11: Diabatic potentials for states $A, B$, and $C$ as a function of $Q_{1}$ at fixed $Q_{2,3,4,5}=0$ (upper panel) and as a function of $Q_{2}$ at fixed $Q_{1,3,4,5}=0$ (lower panel). The red dot indicates the initial position of the wave packet, placed on state $C$. The red thin line marks the energy of the intersection (In).

Finally, we consider the three-state five-dimensional model. The model was introduced by Krause and Matsika to study dynamics at threestate intersections. ${ }^{70}$ To do so, they used the MCTDH method. The population transfer between three states was discussed in the framework of the adiabatic picture.

Since in our work we concentrate on the performance of the CS method, which uses the diabatic propagation of the wave function (similarly to most quantum-dynamical methods), we prefer to compare diabatic populations. The direct diabatic-to-adiabatic transformation 
is very costly, considering dependence on five dimensions. At the same time we want to avoid errors coming from approximate transformation procedures. Thus, we consider the case for which the diabatic MCTDH populations are available. ${ }^{74}$ The parameters corresponding to this particular case are described in 3.2.

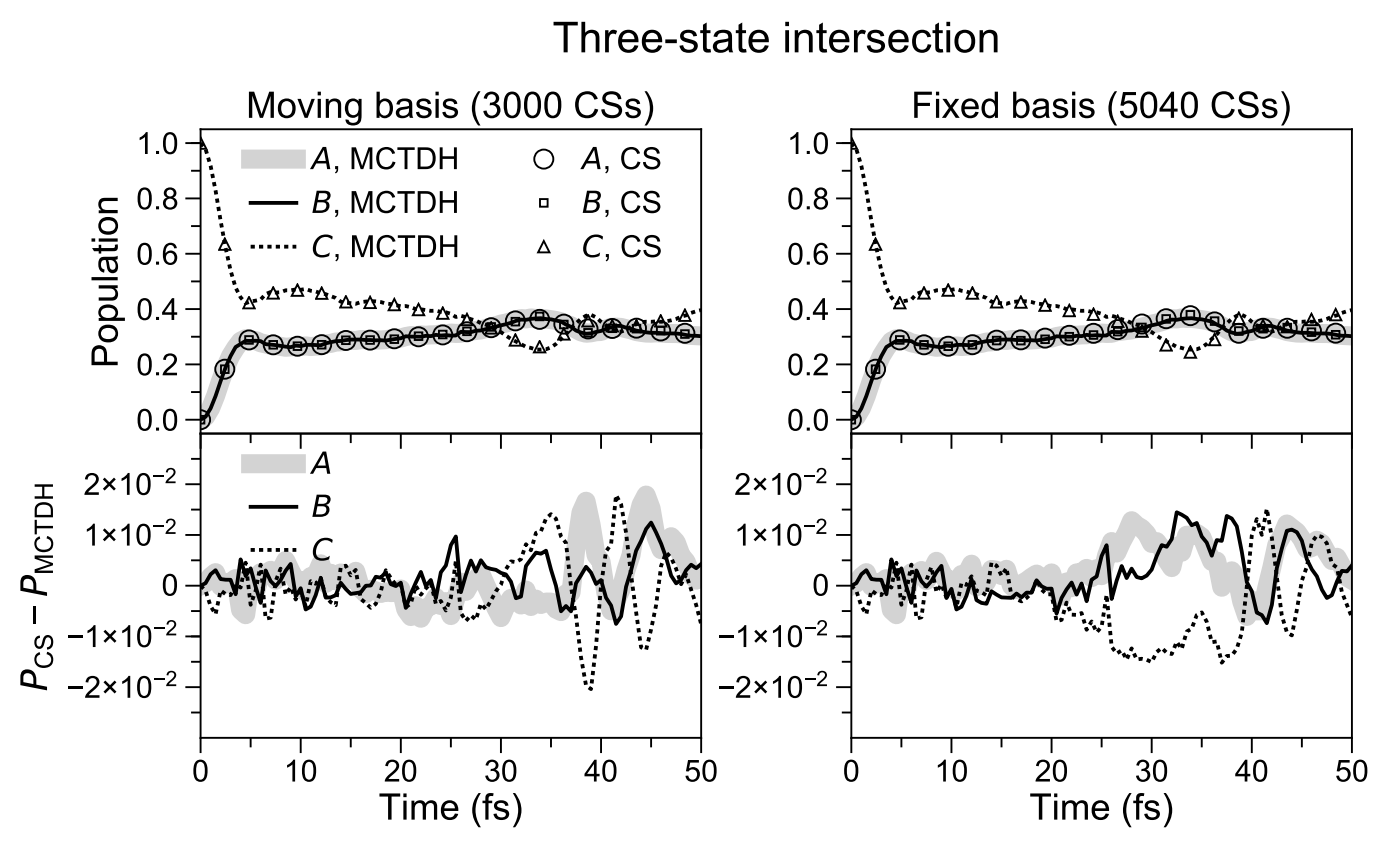

Figure 12: Diabatic populations obtained with the moving and fixed CS basis sets, compared to MCTDH results, for the initial wave packet centered at $\left(Q_{1}, Q_{2}, Q_{3}, Q_{4}, Q_{5}\right)=(0,0,0,0,0)$ and located on state $C$. The differences between coherent state and $\mathrm{MCTDH}$ populations, $P_{\mathrm{CS}}-P_{\mathrm{MCTDH}}$, are also shown.

The cross sections of diabatic potentials together with the initial position of the wave packet and the energy of the three-state intersection are shown in Fig. 11. In this case the initial wave packet is placed directly at the intersection $\left(Q_{1}, Q_{2}, Q_{3}, Q_{4}, Q_{5}\right)=(0,0,0,0,0)$, and assigned to state $C$. We used $\gamma=1$ for all nuclear DoFs, what again corresponds to the width of the initial wave packet.

The diabatic populations computed with the CS method using both moving and fixed basis sets are presented in Fig. 12. The reference MCTDH populations are also shown in Fig. 12. A very good agreement between the CS and MCTDH populations is achieved. Quantitatively, 
the population differences $P_{C S}-P_{M C T D H}$ are on the order of $10^{-2}$ for both basis types.

In the present example, $3000 \mathrm{CSs}$ were used in the case of the moving basis and $5040 \mathrm{CSs}$ in the case of the fixed basis. The initial CSs were sampled from the corresponding Wigner function, i.e. from $W(\mathbf{Q}, \mathbf{P})$, for the moving-basis propagation and from a more compact distribution, $W^{5}(\mathbf{Q}, \mathbf{P})$, for the fixed basis set. The latter was found to yield better agreement with the MCTDH reference results in the case of the fixed-basis approach. Here we have not used the QR decomposition, since the smooth propagation yielding reasonable results was achieved without this procedure. This is due to the larger dimensionality of the nuclear phase space. For the fixed basis, 240 trajectories were propagated for $2060 \mathrm{au}$ ( $\sim 50 \mathrm{fs}$ ) and the Gaussians were sampled with a period of $100 \mathrm{au}(\sim 2.419 \mathrm{fs})$, thus resulting in 5040 fixed basis CSs.

\subsection{Note on computational cost}

The moving basis approach requires to recompute the matrix elements of Hamiltonian, overlap and overlap with derivative matrices with the time step used for trajectory propagation (see (20) and appendix B), since the basis is updated with this time step and, hence, all the mentioned matrices change. Moreover, the time needed to perform the rank-revealing QR decomposition (to select initial coherent state basis functions) for the large matrices, e.g. $20,000 \times 20,000$, is substantial.

For the fixed basis, on the contrary, the Hamiltonian and overlap matrices should be computed only once, in the beginning (if memory permits to store them throughout the simulation), and, obviously, the overlap with the derivative matrix does not enter the equations (see (21) and appendix A). The drawback, however, is the expectedly large number of coherent states used. This leads to larger matrices, and thus slower solution of matrix equations of the type $\mathbf{M a}=\mathbf{c}$.

In our calculations, the propagation with the fixed basis set was found to be up to a factor of $\sim 10$ faster than the propagation with the moving basis set. 


\section{Conclusions}

We have compared the performance of moving and fixed basis sets used with the MCE-type quantum dynamics method for the cases of two- and three-state (conical) intersections of potential energy surfaces. The moving basis sets are usually employed in the Gaussian wave packet approaches to the modeling of quantum dynamics, while the fixed basis sets, sampled from the trajectories, represent a rather new, promising alternative.

We have demonstrated that the both basis set types allow one to achieve a highly accurate description of the population transfer in the regions of two- and three-state (conical) intersections, as being judged by direct comparison with the reference quantum dynamics results, dynamics on a grid for the two-state two-dimensional model and MCTDH dynamics for the three-state five-dimensional case.

Through detailed investigation of the wave packet time evolutions for the two-state two-dimensional model, we conclude that for this model the fixed basis sets outperform the moving ones in the description of true nonlocal spreading of the wave packet and better reproduce the long time dynamics, yielding the wave packets almost identical to the references. Moreover, for the models investigated in the present work, the computational time spent to obtain high accuracy results for population transfer dynamics is much smaller when the fixed basis set is used.

An assessment of the two flavors of basis sets for highly multidimensional systems with a help of more sophisticated techniques for control of the size and quality of the basis set (e.g., basis function cloning and generation of adaptive grids) is of interest for future research.

\section{Supporting information}

See supporting information for the detailed comparison of wave packets obtained with different approaches.

\section{Acknowledgements}

E.T and R.M. are grateful to the Deutsche Forschungsgemeinschaft (DFG) for financial support through "FOR 1809: Light-induced Dynamics in Molecular Aggregates". A.H. and R.M. acknowledge funding 
by the European Research Council (ERC) Consolidator Grant DYNAMO (Grant No. 646737). E.T. thanks Dr. Pascal Krause for valuable discussions and providing MCTDH diabatic populations.

\section{Appendix. Integration of expansion co- efficients}

\section{A Fixed basis set}

First, we consider the case of the time-independent basis. In order to propagate expansion coefficients of (21), one should solve the equation of the form (given below for the case of three electronic states, as an example):

$$
\dot{\mathbf{c}}^{(A)}(t)=\mathbf{F}_{A}\left(\mathbf{a}^{(A)}(t), \mathbf{a}^{(B)}(t), \mathbf{a}^{(C)}(t)\right)
$$

where coefficients $\mathbf{c}$ are related to coefficients a via the overlap matrix $\mathbf{M}(\operatorname{see}(15))$ :

$$
\mathbf{c}^{(A)}(t)=\mathbf{M a}^{(A)}(t)
$$

The exact form of the $\mathbf{F}_{A}$ function is given in (21). Equation (A.1) differs from the standard problem treated with the Runge-Kutta integration scheme in that different coefficients enter the left-hand side and the right-hand side of the equation.

To integrate (A.1) we use the following procedure. The coefficients $\mathbf{c}^{(A)}$ at time $t_{0}+\Delta t$ are found as in the classical Runge-Kutta method:

$$
\mathbf{c}^{(A)}\left(t_{0}+\Delta t\right)=\mathbf{c}^{(A)}\left(t_{0}\right)+\frac{\Delta t}{6}\left(\mathbf{k}_{1}^{(A)}+2 \mathbf{k}_{2}^{(A)}+2 \mathbf{k}_{3}^{(A)}+\mathbf{k}_{4}^{(A)}\right)
$$

Here,

$$
\mathbf{k}_{1}^{(A)}=\mathbf{F}_{A}\left(\mathbf{a}^{(A)}\left(t_{0}\right), \mathbf{a}^{(B)}\left(t_{0}\right), \mathbf{a}^{(C)}\left(t_{0}\right)\right)
$$

The $\mathbf{k}_{2}^{(A)}$ term, in turn, should be computed using coefficients $\mathbf{a}_{1}$ corresponding to

$$
\mathbf{c}^{(A)}\left(t_{0}\right)+\frac{\Delta t}{2} \mathbf{k}_{1}^{(A)}
$$

These $\mathbf{a}_{1}$ coefficients can be obtained solving matrix equation

$$
\mathbf{M a}_{1}^{(A)}=\mathbf{c}^{(A)}\left(t_{0}\right)+\frac{\Delta t}{2} \mathbf{k}_{1}^{(A)}
$$


Then

$$
\mathbf{k}_{2}^{(A)}=\mathbf{F}_{A}\left(\mathbf{a}_{1}^{(A)}, \mathbf{a}_{1}^{(B)}, \mathbf{a}_{1}^{(C)}\right)
$$

Analogously,

$$
\mathbf{k}_{3}^{(A)}=\mathbf{F}_{A}\left(\mathbf{a}_{2}^{(A)}, \mathbf{a}_{2}^{(B)}, \mathbf{a}_{2}^{(C)}\right)
$$

where $\mathbf{a}_{2}$ is a solution to the matrix equation:

$$
\mathbf{M a}_{2}^{(A)}=\mathbf{c}^{(A)}\left(t_{0}\right)+\frac{\Delta t}{2} \mathbf{k}_{2}^{(A)}
$$

And

$$
\mathbf{k}_{4}^{(A)}=\mathbf{F}_{A}\left(\mathbf{a}_{3}^{(A)}, \mathbf{a}_{3}^{(B)}, \mathbf{a}_{3}^{(C)}\right)
$$

with $\mathbf{a}_{3}$ being a solution to

$$
\mathbf{M a}_{3}^{(A)}=\mathbf{c}^{(A)}\left(t_{0}\right)+\Delta t \mathbf{k}_{3}^{(A)}
$$

Finally, the new a coefficients, i.e. at time $t_{0}+\Delta t$, are obtained as a solution to

$$
\mathbf{M a}^{(A)}\left(t_{0}+\Delta t\right)=\mathbf{c}^{(A)}\left(t_{0}+\Delta t\right)
$$

\section{B Moving basis set}

For the case of the moving basis set, see eq. (20), we modify the presented algorithm as follows. Now we should solve

$$
\dot{\mathbf{d}}^{(A)}(t)=\mathbf{G}_{A}\left(\mathbf{b}^{(A)}(t), \mathbf{b}^{(B)}(t), \mathbf{b}^{(C)}(t)\right)
$$

The relation between $\mathbf{d}$ and $\mathbf{b}$ coefficients is (see eqs. (18) and (15))

$$
\mathbf{d}^{(A)}(t) \odot \exp \left(\frac{\mathrm{i}}{\hbar} \mathbf{S}^{(A)}(t)\right)=\mathbf{M}(t)\left[\mathbf{b}^{(A)}(t) \odot \exp \left(\frac{\mathrm{i}}{\hbar} \mathbf{S}^{(A)}(t)\right)\right]
$$

Here $\odot$ denotes the element-wise multiplication of two vectors. The exact form of $\mathbf{G}_{A}$ is given in (20). Again, the new $\mathbf{d}$ coefficients, at time $t_{0}+\Delta t$ are found as

$$
\mathbf{d}^{(A)}\left(t_{0}+\Delta t\right)=\mathbf{d}^{(A)}\left(t_{0}\right)+\frac{\Delta t}{6}\left(\mathbf{k}_{1}^{(A)}+2 \mathbf{k}_{2}^{(A)}+2 \mathbf{k}_{3}^{(A)}+\mathbf{k}_{4}^{(A)}\right)
$$

First,

$$
\mathbf{k}_{1}^{(A)}=\mathbf{G}_{A}\left(\mathbf{b}^{(A)}\left(t_{0}\right), \mathbf{b}^{(B)}\left(t_{0}\right), \mathbf{b}^{(C)}\left(t_{0}\right)\right)
$$


Then, in order to find $\mathbf{k}_{2}^{(A)}$, we should calculate the $\mathbf{b}_{1}$ coefficients corresponding to

$$
\mathbf{d}^{(A)}\left(t_{0}\right)+\frac{\Delta t}{2} \mathbf{k}_{1}^{(A)}
$$

To obtain these $\mathbf{b}_{1}$ coefficients we solve the following matrix equation:

$\mathbf{M}\left(t_{0}+\frac{\Delta t}{2}\right)\left[\mathbf{b}_{1}^{(A)} \odot \exp \left(\frac{\mathrm{i}}{\hbar} \mathbf{S}^{(A)}\left(t_{0}+\frac{\Delta t}{2}\right)\right)\right]=\left(\mathbf{d}^{(A)}\left(t_{0}\right)+\frac{\Delta t}{2} \mathbf{k}_{1}^{(A)}\right) \odot \exp \left(\frac{\mathrm{i}}{\hbar} \mathbf{S}^{(A)}\left(t_{0}+\frac{\Delta t}{2}\right)\right)$

Here, we see that the overlap matrix as well as the action should be known at time $t_{0}+\frac{\Delta t}{2}$, to solve this matrix equation. Thus, the time step for propagation of trajectories should be $\frac{\Delta t}{2}$, i.e. two times smaller than the time step for propagation of coefficients. Then,

$$
\mathbf{k}_{2}^{(A)}=\mathbf{G}_{A}\left(\mathbf{b}_{1}^{(A)}, \mathbf{b}_{1}^{(B)}, \mathbf{b}_{1}^{(C)}\right)
$$

Next,

$$
\mathbf{k}_{3}^{(A)}=\mathbf{G}_{A}\left(\mathbf{b}_{2}^{(A)}, \mathbf{b}_{2}^{(B)}, \mathbf{b}_{2}^{(C)}\right)
$$

where $\mathbf{b}_{2}$ is a solution to the matrix equation

$\mathbf{M}\left(t_{0}+\frac{\Delta t}{2}\right)\left[\mathbf{b}_{2}^{(A)} \odot \exp \left(\frac{\mathrm{i}}{\hbar} \mathbf{S}^{(A)}\left(t_{0}+\frac{\Delta t}{2}\right)\right)\right]=\left(\mathbf{d}^{(A)}\left(t_{0}\right)+\frac{\Delta t}{2} \mathbf{k}_{2}^{(A)}\right) \odot \exp \left(\frac{\mathrm{i}}{\hbar} \mathbf{S}^{(A)}\left(t_{0}+\frac{\Delta t}{2}\right)\right)$

And

$$
\mathbf{k}_{4}^{(A)}=\mathbf{G}_{A}\left(\mathbf{b}_{3}^{(A)}, \mathbf{b}_{3}^{(B)}, \mathbf{b}_{3}^{(C)}\right)
$$

with $\mathbf{b}_{3}$ being a solution to

$$
\mathbf{M}\left(t_{0}+\Delta t\right)\left[\mathbf{b}_{3}^{(A)} \odot \exp \left(\frac{\mathrm{i}}{\hbar} \mathbf{S}^{(A)}\left(t_{0}+\Delta t\right)\right)\right]=\left(\mathbf{d}^{(A)}\left(t_{0}\right)+\Delta t \mathbf{k}_{3}^{(A)}\right) \odot \exp \left(\frac{\mathrm{i}}{\hbar} \mathbf{S}^{(A)}\left(t_{0}+\Delta t\right)\right)
$$

Finally, the new $\mathbf{b}$ coefficients, i.e. at time $t_{0}+\Delta t$, are obtained as a solution to

$$
\mathbf{M}\left(t_{0}+\Delta t\right)\left[\mathbf{b}^{(A)}\left(t_{0}+\Delta t\right) \odot \exp \left(\frac{\mathrm{i}}{\hbar} \mathbf{S}^{(A)}\left(t_{0}+\Delta t\right)\right)\right]=\mathbf{d}^{(A)}\left(t_{0}+\Delta t\right) \odot \exp \left(\frac{\mathrm{i}}{\hbar} \mathbf{S}^{(A)}\left(t_{0}+\Delta t\right)\right)
$$




\section{References}

1. G. Herzberg, H. C. Longuet-Higgins, Intersection of potential energy surfaces in polyatomic molecules, Discuss. Faraday Soc. 35 (1963) 77-82. doi:10.1039/DF9633500077.

2. H. C. Longuet-Higgins, The intersection of potential energy surfaces in polyatomic molecules, Proc. R. Soc. A 344 (1637) (1975) 147-156. doi:10.1098/rspa.1975.0095.

3. H. Köppel, L. S. Cederbaum, W. Domcke, S. S. Shaik, Symmetry breaking and non-born-oppenheimer effects in radical cations, Angew. Chem. Int. Ed. 22 (3) (1983) 210-224. doi:10.1002/ anie. 198302101.

4. A. L. Sobolewski, W. Domcke, C. Dedonder-Lardeux, C. Jouvet, Excited-state hydrogen detachment and hydrogen transfer driven by repulsive $1 \pi \sigma^{*}$ states: A new paradigm for nonradiative decay in aromatic biomolecules, Phys. Chem. Chem. Phys. 4 (2002) 1093-1100. doi:10.1039/B110941N.

URL http://dx.doi.org/10.1039/B110941N

5. U. Werner, R. Mitrić, T. Suzuki, V. Bonačić-Koutecký, Nonadiabatic dynamics within the time dependent density functional theory: Ultrafast photodynamics in pyrazine, Chem. Phys. 349 (1) (2008) 319 - 324. doi:10.1016/j.chemphys.2008.02.061.

6. Galbraith M. C. E., Scheit S., Golubev N. V., Reitsma G., Zhavoronkov N., Despré V., Lépine F., Kuleff A. I., Vrakking M. J. J., Kornilov O., Köppel H., Mikosch J., Few-femtosecond passage of conical intersections in the benzene cation, Nat. Commun. 8 (1) (2017) 1018. doi:10.1038/s41467-017-01133-y.

7. A. H. Zewail, Femtochemistry: Atomic-scale dynamics of the chemical bond, J. Phys. Chem. A 104 (24) (2000) 5660-5694. doi:10.1021/jp001460h.

8. D. Polli, P. Altoè, O. Weingart, K. M. Spillane, C. Manzoni, D. Brida, G. Tomasello, G. Orlandi, P. Kukura, R. A. Mathies, M. Garavelli, G. Cerullo, Conical intersection dynamics of the primary photoisomerization event in vision, Nature 467 (2010) 440443. doi:10.1038/nature09346. 
9. V. Bonačić-Koutecký, R. Mitrić, Theoretical exploration of ultrafast dynamics in atomic clusters: Analysis and control, Chem. Rev. 105 (1) (2005) 11-66. doi:10.1021/cr0206925.

10. A. M. Müller, S. Lochbrunner, W. E. Schmid, W. Fuß, Lowtemperature photochemistry of previtamin d: A hula-twist isomerization of a triene, Angew. Chem. Int. Ed. 37 (4) (1998) 505-507. doi :10 .1002/(SICI) 1521-3773(19980302) 37:4<505: : AID-ANIE505>3.0.CO;2-U.

11. S. Gozem, H. L. Luk, I. Schapiro, M. Olivucci, Theory and simulation of the ultrafast double-bond isomerization of biological chromophores, Chem. Rev. 117 (22) (2017) 13502-13565. doi:10.1021/acs. chemrev.7b00177.

12. M. S. Schuurman, A. Stolow, Dynamics at conical intersections, Annu. Rev. Phys. Chem. 69 (1) (2018) 427-450. doi:10.1146/ annurev-physchem-052516-050721.

13. S. Hahn, G. Stock, Quantum-mechanical modeling of the femtosecond isomerization in rhodopsin, J. Phys. Chem. B 104 (6) (2000) 1146-1149. doi:10.1021/jp992939g.

14. M. Quick, A. L. Dobryakov, M. Gerecke, C. Richter, F. Berndt, I. N. Ioffe, A. A. Granovsky, R. Mahrwald, N. P. Ernsting, S. A. Kovalenko, Photoisomerization dynamics and pathways of transand cis-azobenzene in solution from broadband femtosecond spectroscopies and calculations, J. Phys. Chem. B 118 (29) (2014) 8756-8771. doi:10.1021/jp504999f.

15. R. Mitrić, U. Werner, V. Bonačić-Koutecký, Nonadiabatic dynamics and simulation of time resolved photoelectron spectra within time-dependent density functional theory: Ultrafast photoswitching in benzylideneaniline, J. Chem. Phys. 129 (16) (2008) 164118. doi:10.1063/1.3000012.

16. E. Titov, G. Granucci, J. P. Götze, M. Persico, P. Saalfrank, Dynamics of azobenzene dimer photoisomerization: Electronic and steric effects, J. Phys. Chem. Lett. 7 (18) (2016) 3591-3596. doi:10.1021/acs.jpclett.6b01401. 
17. G. J. Atchity, S. S. Xantheas, K. Ruedenberg, Potential energy surfaces near intersections, J. Chem. Phys. 95 (3) (1991) 18621876. doi:10.1063/1.461036.

18. W. Domcke, D. R. Yarkony, Role of conical intersections in molecular spectroscopy and photoinduced chemical dynamics, Annu. Rev. Phys. Chem. 63 (1) (2012) 325-352. doi:10.1146/ annurev-physchem-032210-103522.

19. W. Domcke, D. R. Yarkony, H. Köppel, Conical Intersections, WORLD SCIENTIFIC, 2004. doi:10.1142/5406.

20. W. Domcke, D. R. Yarkony, H. Köppel, Conical Intersections, WORLD SCIENTIFIC, 2011. doi:10.1142/7803.

21. J. Katriel, E. Davidson, The non-crossing rule: triply degenerate ground-state geometries of ch4+, Chem. Phys. Lett. 76 (2) (1980) 259 - 262. doi:10.1016/0009-2614(80)87016-3.

22. S. Matsika, D. R. Yarkony, Accidental conical intersections of three states of the same symmetry. i. location and relevance, J. Chem. Phys. 117 (15) (2002) 6907-6910. doi:10.1063/1.1513304.

23. S. Matsika, D. R. Yarkony, Beyond two-state conical intersections. three-state conical intersections in low symmetry molecules: The allyl radical, J. Am. Chem. Soc. 125 (35) (2003) 10672-10676. doi:10.1021/ja036201v.

24. S. Matsika, D. R. Yarkony, Conical intersections of three electronic states affect the ground state of radical species with little or no symmetry: Pyrazolyl, J. Am. Chem. Soc. 125 (41) (2003) 1242812429. doi:10.1021/ja037925+.

25. J. C. Tully, Perspective: Nonadiabatic dynamics theory, J. Chem. Phys. 137 (22) (2012) 22A301. doi:10.1063/1.4757762.

26. B. F. E. Curchod, T. J. Martínez, Ab initio nonadiabatic quantum molecular dynamics, Chem. Rev. 118 (7) (2018) 3305-3336. doi: 10.1021/acs . chemrev. 7 b00423.

27. R. Crespo-Otero, M. Barbatti, Recent advances and perspectives on nonadiabatic mixed quantum-classical dynamics, Chem. Rev. 118 (15) (2018) 7026-7068. doi:10.1021/acs.chemrev.7b00577. 
28. J. Albert, D. Kaiser, V. Engel, Communication: Adiabatic and non-adiabatic electron-nuclear motion: Quantum and classical dynamics, J. Chem. Phys. 144 (17) (2016) 171103. doi:10.1063/1. 4948777.

29. J. C. Tully, Molecular dynamics with electronic transitions, J. Chem. Phys. 93 (2) (1990) 1061-1071. doi:10.1063/1.459170.

30. M. Persico, G. Granucci, An overview of nonadiabatic dynamics simulations methods, with focus on the direct approach versus the fitting of potential energy surfaces, Theor. Chem. Acc. 133 (9) (2014) 1526. doi:10.1007/s00214-014-1526-1.

31. H.-D. Meyer, U. Manthe, L. Cederbaum, The multiconfigurational time-dependent hartree approach, Chem. Phys. Lett. 165 (1) (1990) 73 - 78. doi:10.1016/0009-2614(90) 87014-I.

32. M. Beck, A. Jäckle, G. Worth, H.-D. Meyer, The multiconfiguration time-dependent Hartree (MCTDH) method: a highly efficient algorithm for propagating wavepackets, Phys. Rep. 324 (1) (2000) 1-105. doi:10.1016/S0370-1573(99)00047-2.

33. H. Wang, M. Thoss, Multilayer formulation of the multiconfiguration time-dependent hartree theory, J. Chem. Phys. 119 (3) (2003) 1289-1299. doi:10.1063/1.1580111.

34. U. Manthe, A multilayer multiconfigurational time-dependent hartree approach for quantum dynamics on general potential energy surfaces, J. Chem. Phys. 128 (16) (2008) 164116. doi: $10.1063 / 1.2902982$.

35. E. J. Heller, Frozen gaussians: A very simple semiclassical approximation, J. Chem. Phys. 75 (6) (1981) 2923-2931. doi: $10.1063 / 1.442382$.

36. I. Burghardt, H.-D. Meyer, L. S. Cederbaum, Approaches to the approximate treatment of complex molecular systems by the multiconfiguration time-dependent hartree method, J. Chem. Phys. 111 (7) (1999) 2927-2939. doi:10.1063/1.479574. 
37. I. Burghardt, K. Giri, G. A. Worth, Multimode quantum dynamics using gaussian wavepackets: The gaussian-based multiconfiguration time-dependent hartree (g-mctdh) method applied to the absorption spectrum of pyrazine, J. Chem. Phys. 129 (17) (2008) 174104. doi:10.1063/1.2996349.

38. G. Richings, I. Polyak, K. Spinlove, G. Worth, I. Burghardt, B. Lasorne, Quantum dynamics simulations using gaussian wavepackets: the vmcg method, Int. Rev. Phys. Chem. 34 (2) (2015) 269 308. doi:10.1080/0144235X.2015.1051354.

39. T. J. Martinez, M. Ben-Nun, R. D. Levine, Multi-electronicstate molecular dynamics: A wave function approach with applications, J. Phys. Chem. 100 (19) (1996) 7884-7895. doi: 10.1021/jp953105a.

40. M. Ben-Nun, T. J. Martinez, Nonadiabatic molecular dynamics: Validation of the multiple spawning method for a multidimensional problem, J. Chem. Phys. 108 (17) (1998) 7244-7257. doi:10. 1063/1.476142.

41. M. Ben-Nun, J. Quenneville, T. J. Martínez, Ab initio multiple spawning: Photochemistry from first principles quantum molecular dynamics, J. Phys. Chem. A 104 (22) (2000) 5161-5175. doi:10.1021/jp994174i.

42. D. V. Shalashilin, Quantum mechanics with the basis set guided by ehrenfest trajectories: Theory and application to spin-boson model, J. Chem. Phys. 130 (24) (2009) 244101. doi:10.1063/1. 3153302 .

43. D. V. Shalashilin, Nonadiabatic dynamics with the help of multiconfigurational ehrenfest method: Improved theory and fully quantum 24d simulation of pyrazine, J. Chem. Phys. 132 (24) (2010) 244111. doi:10.1063/1.3442747.

44. K. Saita, D. V. Shalashilin, On-the-fly ab initio molecular dynamics with multiconfigurational ehrenfest method, J. Chem. Phys. 137 (22) (2012) 22A506. doi:10.1063/1.4734313.

45. S. Fernandez-Alberti, D. V. Makhov, S. Tretiak, D. V. Shalashilin, Non-adiabatic excited state molecular dynamics of pheny- 
lene ethynylene dendrimer using a multiconfigurational ehrenfest approach, Phys. Chem. Chem. Phys. 18 (2016) 10028-10040. doi:10.1039/C5CP07332D.

46. I. Horenko, C. Salzmann, B. Schmidt, C. Schütte, Quantumclassical liouville approach to molecular dynamics: Surface hopping gaussian phase-space packets, J. Chem. Phys. 117 (24) (2002) 11075-11088. doi:10.1063/1.1522712.

47. A. Humeniuk, R. Mitrić, Non-adiabatic dynamics around a conical intersection with surface-hopping coupled coherent states, J. Chem. Phys. 144 (23) (2016) 234108. doi:10.1063/1.4954189.

48. D. V. Makhov, W. J. Glover, T. J. Martinez, D. V. Shalashilin, $\mathrm{Ab}$ initio multiple cloning algorithm for quantum nonadiabatic molecular dynamics, J. Chem. Phys. 141 (5) (2014) 054110. doi: $10.1063 / 1.4891530$.

49. V. M. Freixas, S. Fernandez-Alberti, D. V. Makhov, S. Tretiak, D. Shalashilin, An ab initio multiple cloning approach for the simulation of photoinduced dynamics in conjugated molecules, Phys. Chem. Chem. Phys. 20 (2018) 17762-17772. doi:10.1039/ C8CP02321B.

50. C. W. Heaps, D. A. Mazziotti, Accurate non-adiabatic quantum dynamics from pseudospectral sampling of time-dependent gaussian basis sets, J. Chem. Phys. 145 (6) (2016) 064101. doi: $10.1063 / 1.4959872$.

51. A. J. Jenkins, K. E. Spinlove, M. Vacher, G. A. Worth, M. A. Robb, The ehrenfest method with fully quantum nuclear motion (qu-eh): Application to charge migration in radical cations, J. Chem. Phys. 149 (9) (2018) 094108. doi:10.1063/1.5038428.

52. L. Chen, M. F. Gelin, W. Domcke, Multimode quantum dynamics with multiple davydov d2 trial states: Application to a 24dimensional conical intersection model, J. Chem. Phys. 150 (2) (2019) 024101. doi:10.1063/1.5066022.

53. P. A. Sherratt, D. V. Shalashillin, M. S. Child, Description of multidimensional tunnelling with the help of coupled coherent states guided by classical hamiltonians with quantum corrections, Chem. 
Phys. 322 (1) (2006) 127 - 134. doi:10.1016/j.chemphys. 2005. 06.050 .

54. R. Martinazzo, M. Nest, P. Saalfrank, G. F. Tantardini, A local coherent-state approximation to system-bath quantum dynamics, J. Chem. Phys. 125 (19) (2006) 194102. doi:10.1063/1.2362821.

55. M. A. C. Saller, S. Habershon, Basis set generation for quantum dynamics simulations using simple trajectory-based methods, J. Chem. Theory Comput. 11 (1) (2015) 8-16. doi:10.1021/ ct500657f.

56. C. Symonds, J. A. Kattirtzi, D. V. Shalashilin, The effect of sampling techniques used in the multiconfigurational Ehrenfest method, J. Chem. Phys. 148 (18) (2018) 184113. doi:10.1063/ 1.5020567 .

57. L. Joubert-Doriol, A. F. Izmaylov, Nonadiabatic quantum dynamics with frozen-width gaussians, J. Phys. Chem. A 122 (29) (2018) 6031-6042. doi:10.1021/acs.jpca.8b03404.

58. A. Kirrander, D. V. Shalashilin, Quantum dynamics with fermion coupled coherent states: Theory and application to electron dynamics in laser fields, Phys. Rev. A 84 (2011) 033406. doi: 10.1103/PhysRevA.84.033406.

59. D. V. Shalashilin, M. S. Child, The phase space ccs approach to quantum and semiclassical molecular dynamics for highdimensional systems, Chem. Phys. 304 (1) (2004) 103-120. doi: $10.1016 / j$. chemphys . 2004.06.013.

60. D. V. Shalashilin, I. Burghardt, Gaussian-based techniques for quantum propagation from the time-dependent variational principle: Formulation in terms of trajectories of coupled classical and quantum variables, J. Chem. Phys. 129 (8) (2008) 084104. doi:10.1063/1.2969101.

61. C. Gerry, P. Knight, Coherent states, Cambridge University Press, 2004, p. 43-73. doi:10.1017/CB09780511791239.003.

62. Wolfram Research, Inc., Mathematica, Version 11.0.1.0, champaign, IL, 2016. 
63. E. Wigner, On the quantum correction for thermodynamic equilibrium, Phys. Rev. 40 (1932) 749-759. doi:10.1103/PhysRev. 40.749 .

64. S. Kube, C. Lasser, M. Weber, Monte carlo sampling of wigner functions and surface hopping quantum dynamics, J. Comput. Phys. 228 (6) (2009) 1947 - 1962. doi:10.1016/j.jcp.2008.11. 016.

65. D. V. Shalashilin, M. S. Child, Basis set sampling in the method of coupled coherent states: Coherent state swarms, trains, and pancakes, J. Chem. Phys. 128 (5) (2008) 054102. doi:10.1063/ 1.2828509 .

66. W. Koch, T. J. Frankcombe, Basis expansion leaping: A new method to solve the time-dependent Schrödinger equation for molecular quantum dynamics, Phys. Rev. Lett. 110 (2013) 263202. doi:10.1103/PhysRevLett.110.263202.

67. M. A. C. Saller, S. Habershon, Quantum dynamics with shorttime trajectories and minimal adaptive basis sets, J. Chem. Theory Comput. 13 (7) (2017) 3085-3096. doi:10.1021/acs.jctc. $7 \mathrm{~b} 00021$.

68. T. Murakami, T. J. Frankcombe, Non-adiabatic quantum molecular dynamics by the basis expansion leaping multi-configuration gaussian (BEL MCG) method: Multi-set and single-set formalisms, J. Chem. Phys. 150 (14) (2019) 144112. doi:10.1063/ 1.5084749 .

69. Y. Arai, K. Suzuki, M. Kanno, H. Kono, Automatic spatial extension of a time-dependent wavefunction expanded in terms of gaussians: Application to multidimensional tunneling, Chem. Phys. Lett. 708 (2018) 170 - 176. doi:https://doi.org/10.1016/j. cplett.2018.07.022.

70. P. Krause, S. Matsika, Nuclear dynamics for a three-state jahn-teller model system, J. Chem. Phys. 136 (3) (2012) 034110. doi:10.1063/1.3677273.

71. A. Ferretti, G. Granucci, A. Lami, M. Persico, G. Villani, Quantum mechanical and semiclassical dynamics at a conical intersec- 
tion, J. Chem. Phys. 104 (14) (1996) 5517-5527. doi:10.1063/1. 471791.

72. S. Yang, J. D. Coe, B. Kaduk, T. J. Martínez, An "optimal" spawning algorithm for adaptive basis set expansion in nonadiabatic dynamics, J. Chem. Phys. 130 (13) (2009) 134113. doi: 10.1063/1.3103930.

73. F. Agostini, B. F. E. Curchod, When the exact factorization meets conical intersections..., Eur. Phys. J. B 91 (7) (2018) 141. doi: 10.1140/epjb/e2018-90117-6.

74. P. Krause, private communication, 2018. 


\title{
Supporting Information for
}

Comparison of moving and fixed basis sets for nonadiabatic quantum dynamics at conical intersections

\author{
Evgenii Titov, Alexander Humeniuk, Roland Mitrić* \\ Institut für Physikalische und Theoretische Chemie, Julius-Maximilians-Universität Würzburg, \\ Emil-Fischer-Straße 42, 97074 Würzburg, Germany \\ *roland.mitric@uni-wuerzburg.de
}




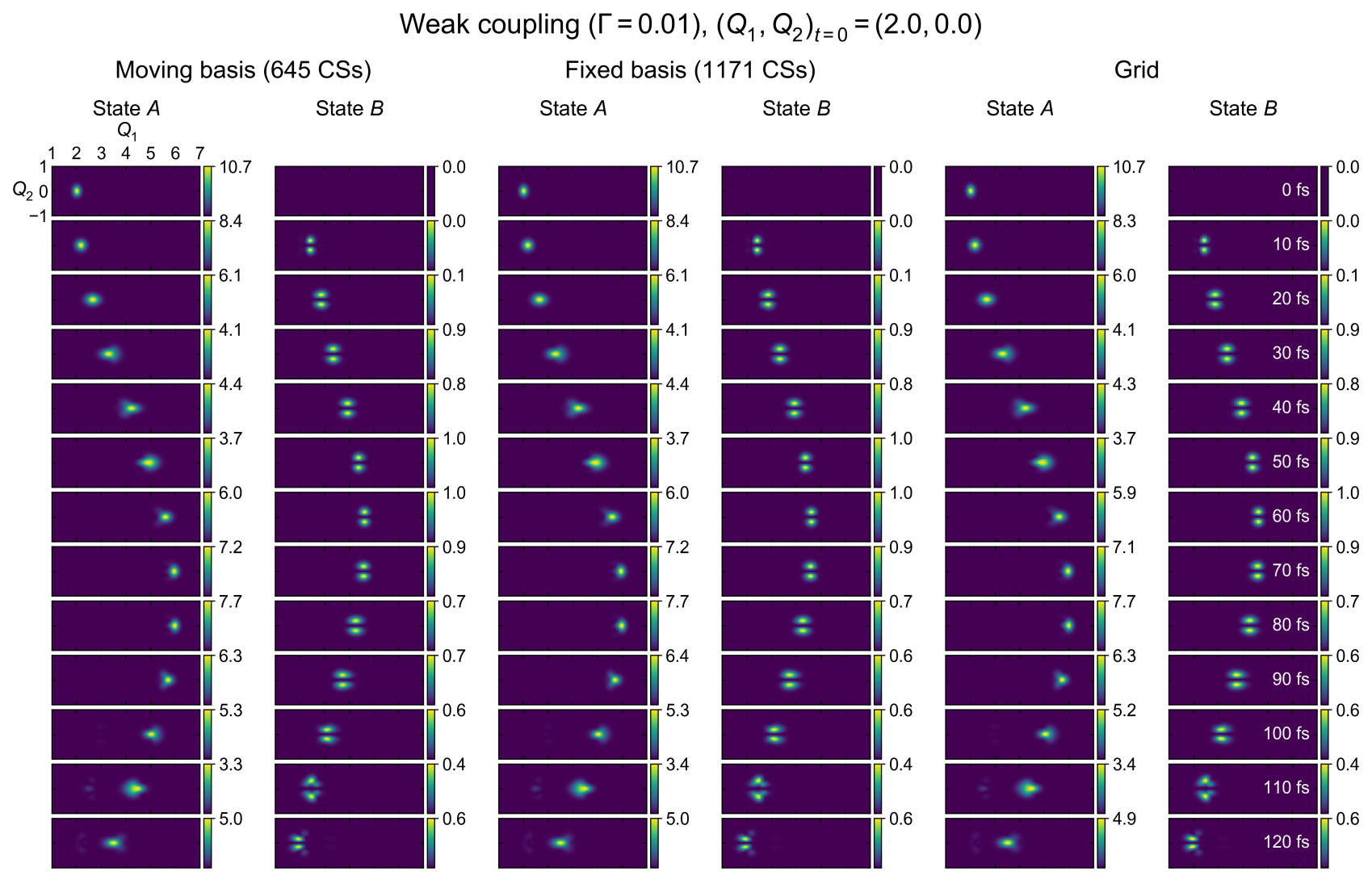

Figure S1: Diabatic wave packet evolution $\left(\left|\psi^{(A)}(\mathbf{Q}, t)\right|^{2}\right.$ and $\left.\left|\psi^{(B)}(\mathbf{Q}, t)\right|^{2}\right)$ for the case of weak coupling $(\Gamma=0.01)$ and the initial position $\left(Q_{1}, Q_{2}\right)=(2.0,0.0)$. Left two columns show the dynamics with 645 moving coherent states, middle two columns show the dynamics with the basis of 1171 fixed coherent states (sampled from 100 Ehrenfest trajectories), right two columns show reference dynamics on a grid. Colorbars span the range from zero to the maximum for a given state at a current time. These maxima are shown as tick labels of colorbars (rounded to a 1st decimal place). The $Q_{1}$ and $Q_{2}$ ranges are the same for all plots and are shown in the left uppermost panel. Time stamps are shown in the last column. 
Strong coupling $(\Gamma=0.08),\left(Q_{1}, Q_{2}\right)_{t=0}=(2.0,0.0)$

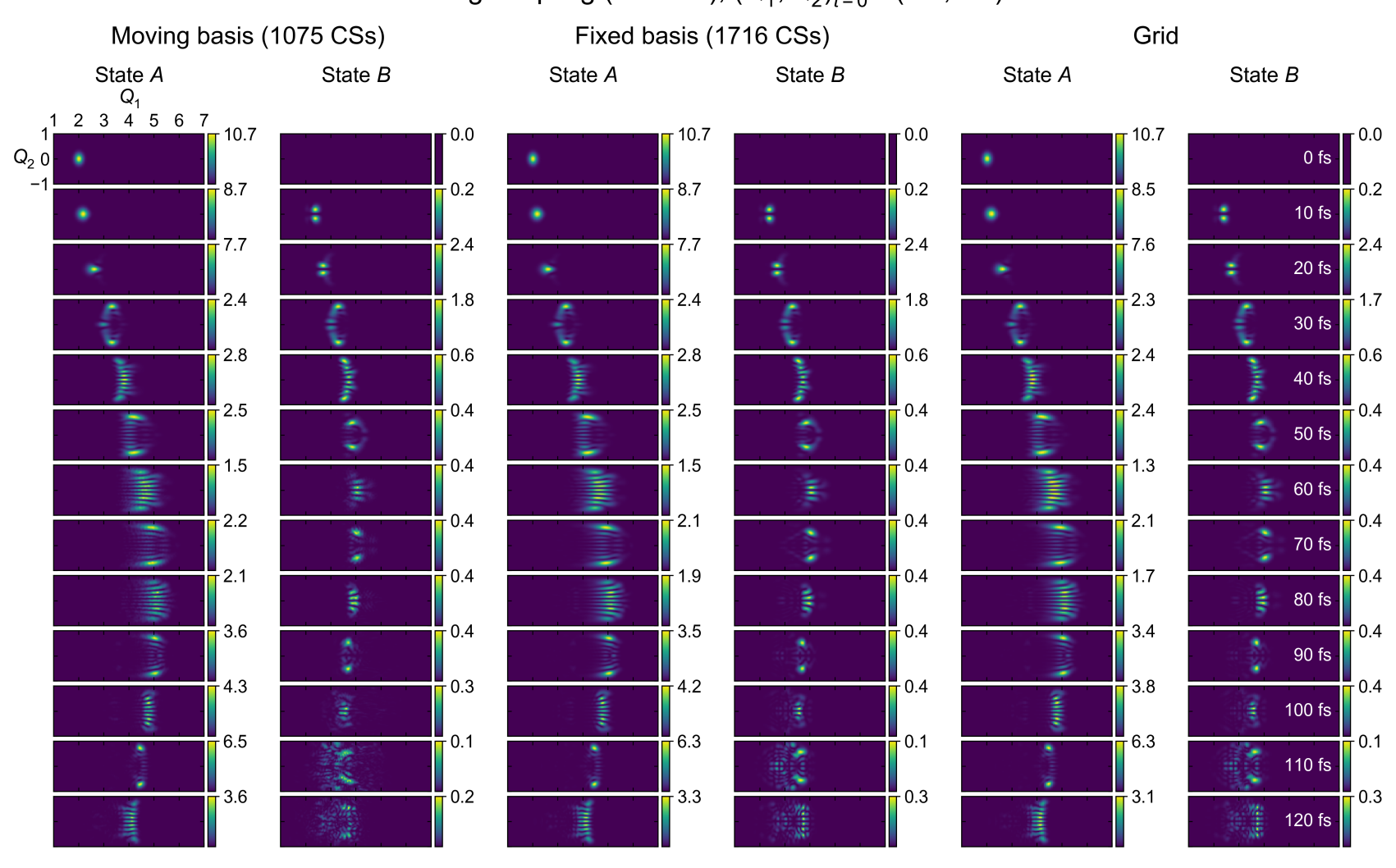

Figure S2: Diabatic wave packet evolution $\left(\left|\psi^{(A)}(\mathbf{Q}, t)\right|^{2}\right.$ and $\left.\left|\psi^{(B)}(\mathbf{Q}, t)\right|^{2}\right)$ for the case of strong coupling $(\Gamma=0.08)$ and the initial position $\left(Q_{1}, Q_{2}\right)=(2.0,0.0)$. Left two columns show the dynamics with 1075 moving coherent states, middle two columns show the dynamics with the basis of 1716 fixed coherent states (sampled from 100 Ehrenfest trajectories), right two columns show reference dynamics on a grid. Colorbars span the range from zero to the maximum for a given state at a current time. These maxima are shown as tick labels of colorbars (rounded to a 1st decimal place). The $Q_{1}$ and $Q_{2}$ ranges are the same for all plots and are shown in the left uppermost panel. Time stamps are shown in the last column. 
Weak coupling $(\Gamma=0.01),\left(Q_{1}, Q_{2}\right)_{t=0}=(5.2,0.0)$

Moving basis (567 CSs)

State $A$

$Q_{1}$
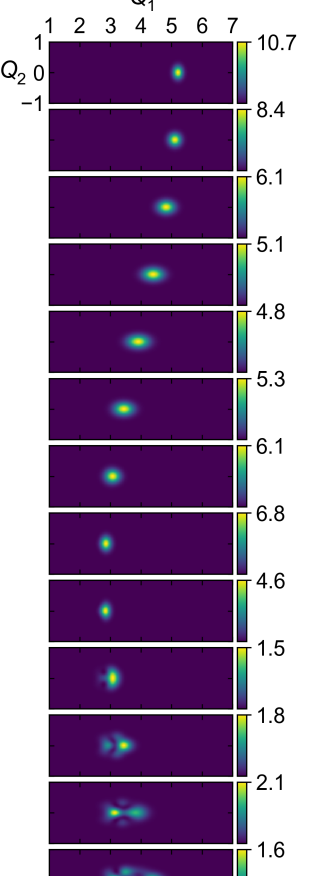

-62*

$x=-1$

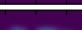

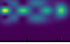

$x-7$

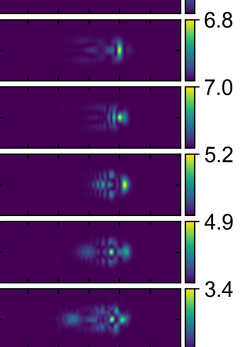

State $B$

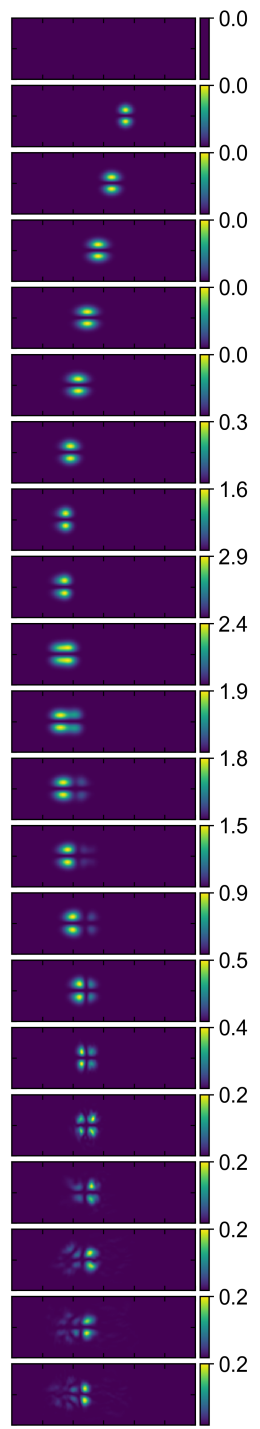

Fixed basis (874 CSs)

State $A$

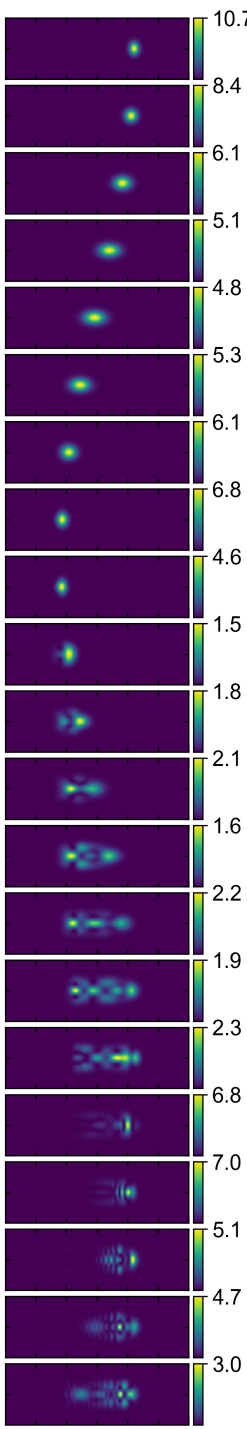

State $B$

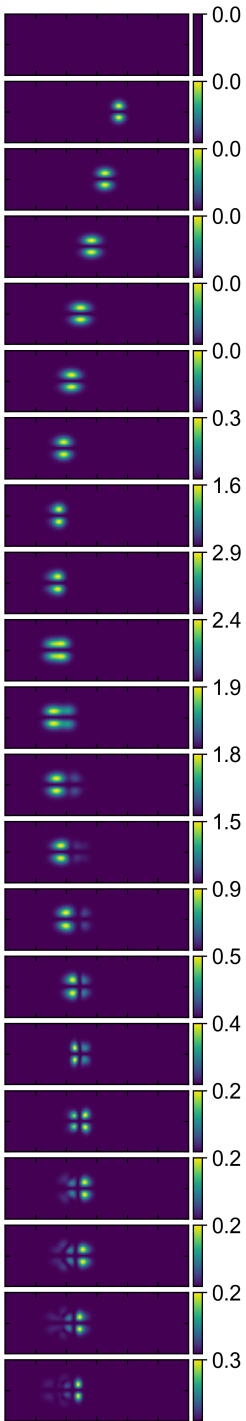

Grid

State $A$

State $B$

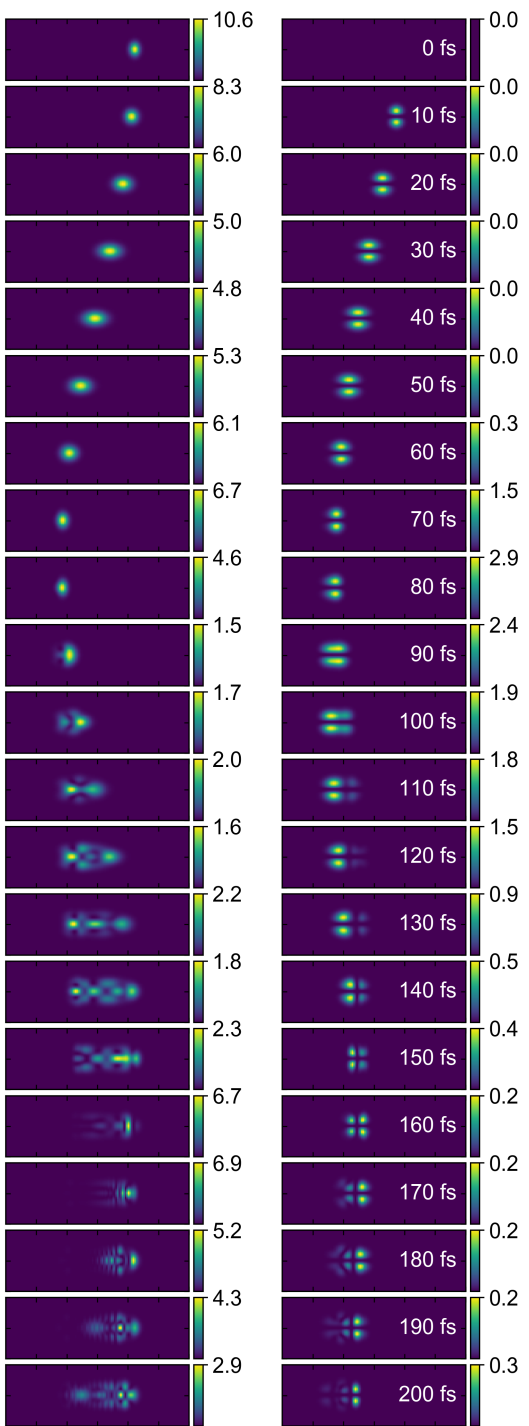

Figure S3: Diabatic wave packet evolution $\left(\left|\psi^{(A)}(\mathbf{Q}, t)\right|^{2}\right.$ and $\left.\left|\psi^{(B)}(\mathbf{Q}, t)\right|^{2}\right)$ for the case of weak coupling $(\Gamma=0.01)$ and the initial position $\left(Q_{1}, Q_{2}\right)=(5.2,0.0)$. Left two columns show the dynamics with 567 moving coherent states, middle two columns show the dynamics with the basis of 874 fixed coherent states (sampled from 100 Ehrenfest trajectories), right two columns show reference dynamics on a grid. Colorbars span the range from zero to the maximum for a given state at a current time. These maxima are shown as tick labels of colorbars (rounded to a 1st decimal place). The $Q_{1}$ and $Q_{2}$ ranges are the same for all plots and are shown in the left uppermost panel. Time stamps are shown in the last column. 
Strong coupling $(\Gamma=0.08),\left(Q_{1}, Q_{2}\right)_{t=0}=(5.2,0.0)$

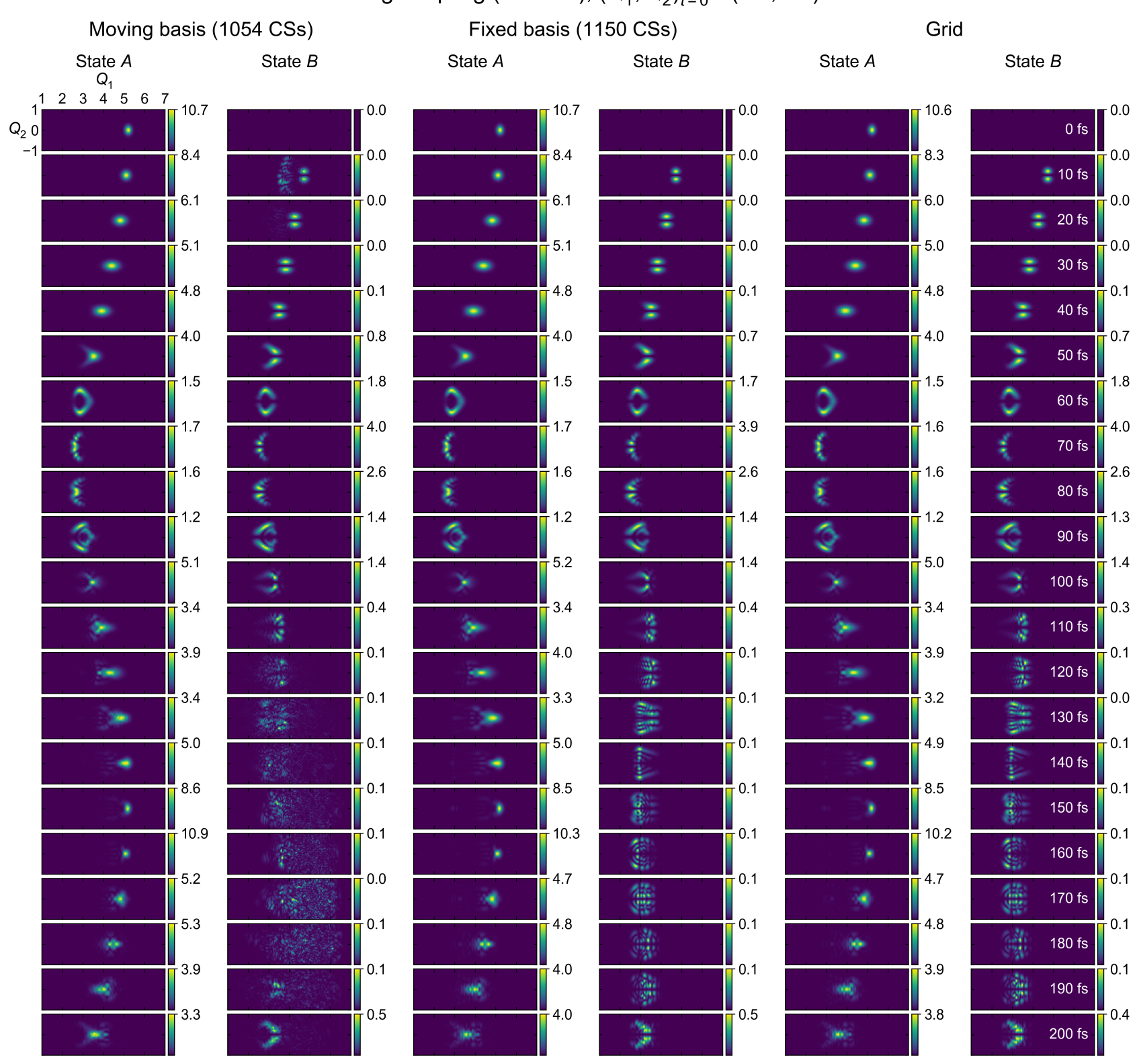

Figure S4: Diabatic wave packet evolution $\left(\left|\psi^{(A)}(\mathbf{Q}, t)\right|^{2}\right.$ and $\left.\left|\psi^{(B)}(\mathbf{Q}, t)\right|^{2}\right)$ for the case of strong coupling $(\Gamma=0.08)$ and the initial position $\left(Q_{1}, Q_{2}\right)=(5.2,0.0)$. Left two columns show the dynamics with 1054 moving coherent states, middle two columns show the dynamics with the basis of 1150 fixed coherent states (sampled from 100 Ehrenfest trajectories), right two columns show reference dynamics on a grid. Colorbars span the range from zero to the maximum for a given state at a current time. These maxima are shown as tick labels of colorbars (rounded to a 1st decimal place). The $Q_{1}$ and $Q_{2}$ ranges are the same for all plots and are shown in the left uppermost panel. Time stamps are shown in the last column. 